\title{
The density of integral points on hypersurfaces of degree at least four
}

by

\section{Oscar Marmon (Gothenburg)}

1. Introduction. Given a polynomial $f \in \mathbb{Z}\left[x_{1}, \ldots, x_{n}\right]$ we wish to study the solutions in $\mathbb{Z}^{n}$ to the Diophantine equation

$$
f\left(x_{1}, \ldots, x_{n}\right)=0 .
$$

We are interested in the density of solutions, that is, for a given positive real number $B$ we want to estimate the number of solutions $\mathbf{x}$ to (1) satisfying $|\mathbf{x}| \leq B$, where $|\mathbf{x}|=\max _{i}\left|x_{i}\right|$. To this end we introduce the counting function

$$
N(f, B)=\#\left\{\mathbf{x} \in \mathbb{Z}^{n} ; f(\mathbf{x})=0,|\mathbf{x}| \leq B\right\} .
$$

We shall use congruences as a tool to estimate $N(f, B)$. Thus, we introduce the counting functions

$$
N(f, B, m)=\#\left\{\mathbf{x} \in \mathbb{Z}^{n} ; f(\mathbf{x}) \equiv 0(\bmod m),|\mathbf{x}| \leq B\right\} .
$$

Trivially, for any $m \in \mathbb{Z}, N(f, B, m)$ is an upper bound for $N(f, B)$. We extend this notation to systems of equations in the obvious way:

$$
\begin{aligned}
N\left(f_{1}, \ldots, f_{r}, B\right)=\#\left\{\mathbf{x} \in \mathbb{Z}^{n} ; f_{1}(\mathbf{x})=\cdots=f_{r}(\mathbf{x})=0,|\mathbf{x}| \leq B\right\}, \\
N\left(f_{1}, \ldots, f_{r}, B, m\right)=\#\left\{\mathbf{x} \in \mathbb{Z}^{n} ;\right. \\
\left.\quad f_{1}(\mathbf{x}) \equiv \cdots \equiv f_{r}(\mathbf{x}) \equiv 0(\bmod m),|\mathbf{x}| \leq B\right\} .
\end{aligned}
$$

By the leading form of the polynomial $f$ we shall mean the homogeneous part of maximal degree. Heath-Brown [12] proved that for a polynomial $f \in \mathbb{Z}\left[X_{1}, \ldots, X_{n}\right]$ of degree at least 3 such that the leading form $F$ is non-singular (i.e. defines a non-singular hypersurface in $\mathbb{P}_{\mathbb{C}}^{n}$ ), we have the estimate

$$
N(f, B) \ll_{F} B^{n-3+15 /(n+5)}
$$

for $n \geq 5$. To prove this, Heath-Brown studied $N(f, B, p q)$ for two different primes $p, q$, and devised a version of van der Corput's method of exponential 
sums as a key step in the estimation of this counting function. By incorporating an exponential sum estimate by Katz [16] into Heath-Brown's method, the author [19] sharpened this result slightly, to

$$
N(f, B) \ll_{F} B^{n-3+(13 n-8) /\left(n^{2}+3 n-2\right)}(\log B)^{n / 2}
$$

for $n \geq 6$. Salberger [20] was able to sharpen the estimate further, through a new geometric argument. He proved

$$
N(f, B) \ll_{F} B^{n-3+9 /(n+2)}(\log B)^{n / 2}
$$

for $n \geq 4$.

For polynomials of degree at least 4, one can try to iterate the Weyl (or van der Corput) differencing step in [12] twice to get even sharper estimates, and that is the approach we will take in this paper. The aim is to prove the following result:

TheOREM 1.1. Let $f$ be a polynomial in $\mathbb{Z}\left[x_{1}, \ldots, x_{n}\right]$ of degree $d \geq 4$ with leading form $F$. Let $Z=\operatorname{Proj} \mathbb{Z}\left[x_{1}, \ldots, x_{n}\right] /(F)$, and suppose that $Z_{\mathbb{Q}}$ is a non-singular subscheme of $\mathbb{P}_{\mathbb{Q}}^{n-1}$. Then we have the estimate

$$
N(f, B) \ll_{F} B^{n-4+(37 n-18) /\left(n^{2}+8 n-4\right)} .
$$

The estimate in Theorem 1.1 improves upon $(2)$ as soon as $n \geq 17$. Moreover, if $n \geq 29$, Theorem 1.1 implies that $N(f, B) \ll_{F} B^{n-3}$.

Using an argument of Heath-Brown, we can derive a uniform version of Theorem 1.1.

THEOREM 1.2. Let $f$ be a polynomial in $\mathbb{Z}\left[x_{1}, \ldots, x_{n}\right]$ of degree $d \geq 4$ with leading form $F$. Let $Z=\operatorname{Proj} \mathbb{Z}\left[x_{1}, \ldots, x_{n}\right] /(F)$, and suppose that $Z_{\mathbb{Q}}$ is a non-singular subscheme of $\mathbb{P}_{\mathbb{Q}}^{n-1}$. Then we have the estimate

$$
N(f, B) \ll_{n, d, \varepsilon} B^{n-4+(37 n-18) /\left(n^{2}+8 n-4\right)}+B^{n-3+\varepsilon}
$$

for any $\varepsilon>0$.

When proving these two theorems, it will be convenient to seek to estimate a weighted counting function rather than the original one. More precisely, let $W: \mathbb{R}^{n} \rightarrow[0,1]$ be an infinitely differentiable function, supported on $[-2,2]^{n}$. Then we define weighted counting functions

$$
N_{W}(f, B, m)=\sum_{\substack{\mathbf{x} \in \mathbb{Z}^{n} \\ m \mid f(\mathbf{x})}} W\left(\frac{1}{B} \mathbf{x}\right)
$$

In the proof of Theorem 1.1 we shall take $W$ to be the function defined by

$$
W(\mathbf{t})=\prod_{i=1}^{n} w\left(t_{i} / 2\right), \quad \text { where } \quad w(t)= \begin{cases}\exp \left(-1 /\left(1-t^{2}\right)\right), & |t|<1 \\ 0, & |t| \geq 1 .\end{cases}
$$


It is then clear that $N(f, B, m) \ll N_{W}(f, B, m)$. Approximating the characteristic function of the cube $[-B, B]^{n}$ with a smooth function in this way allows us to sharpen some of the estimates involved.

The proof of Theorem 1.1 is carried out in Sections 4 and 5 , and incorporates the idea of Salberger (see Remarks 4.1 and 4.2 . We shall use a modulus which is a product of three distinct primes $m=\pi p q$, where the primes $\pi, p$ can be viewed as parameters connected to the two consecutive differencing steps. The two differencings put us in the position to apply results on the density of $\mathbb{F}_{q}$-rational points on a family of new varieties over $\mathbb{F}_{q}$, parameterized by integral $n$-tuples $\mathbf{y}, \mathbf{z}$. These results, behind which lie Deligne's bounds for exponential sums over non-singular varieties, become weaker as the dimensions of the singular loci of the varieties increase, and thus we need to control these dimensions. Section 2 is devoted to this problem.

2. Preliminary geometric results. The geometric arguments in this section extend those of Salberger [20]. A priori, some of our results are valid in characteristic zero only, but in 2.2 we obtain conditions on primes $p$ ensuring the truth of the statements in characteristic $p$.

2.1. Results for polynomials over a field. In this section, suppose that $K$ is a field. Let $\operatorname{char} K=p$. Furthermore, we shall assume that $n \geq 3$.

NotAtion 2.1. If $F \in K\left[x_{1}, \ldots, x_{n}\right]$ is a homogeneous polynomial and $\mathbf{y} \in K^{n}$, we define

$$
F^{\mathbf{y}}(\mathbf{x})=\mathbf{y} \cdot \nabla F(\mathbf{x})=y_{1} \frac{\partial F}{\partial x_{1}}+\cdots+y_{n} \frac{\partial F}{\partial x_{n}} .
$$

Furthermore, for each pair $\mathbf{y}, \mathbf{z}$ of $n$-tuples of elements of $K$, we define

$$
F^{\mathbf{y}, \mathbf{z}}(\mathbf{x})=(\operatorname{Hess} F) \mathbf{y} \cdot \mathbf{z}=\sum_{1 \leq i, j \leq n} \frac{\partial^{2} F}{\partial x_{i} \partial x_{j}} y_{i} z_{j} .
$$

We have $F^{\mathbf{y}, \mathbf{z}}=\left(F^{\mathbf{y}}\right)^{\mathbf{z}}=\left(F^{\mathbf{z}}\right)^{\mathbf{y}}$.

For a collection $F_{1}, \ldots, F_{r}$ of homogeneous polynomials we denote by $V\left(F_{1}, \ldots, F_{r}\right)$ the closed subscheme of $\mathbb{P}_{K}^{n-1}$ that they define. If $F, G$ are two homogeneous polynomials and $\mathbf{z} \in K^{n}$, we define

$$
\operatorname{Diff}_{\mathbf{z}}(F, G)=V\left(F, G, G^{\mathbf{z}}\right) .
$$

The reason for the notation is that the differencing process used in Section 5 will lead us to consider such varieties. Note that the definition is not symmetric in $F$ and $G$.

When $x$ (or any other letter) is used to denote a $K$-point of $\mathbb{P}_{K}^{n-1}$, we will use the corresponding bold letter $\mathbf{x}$ to denote an element of $K^{n}$ representing $x$. Vice versa, given $\mathbf{x} \in K^{n} \backslash\{\mathbf{0}\}$, we denote its homothety class by $x$. 
We denote by $\mathbb{G}(k, n-1)$ the set of $k$-dimensional linear subspaces of $\mathbb{P}_{K}^{n-1}$.

Finally, we adopt the convention that the dimension of the empty variety is -1 .

Definition 2.1. If $V \subset \mathbb{P}_{K}^{n-1}$ is a non-singular hypersurface defined by a homogeneous polynomial $G\left(x_{1}, \ldots, x_{n}\right)$ of degree $d \geq 2$, then the Gauss morphism $\mathcal{G}: V \rightarrow \mathbb{P}_{K}^{n-1}$ is defined by $x \mapsto[\nabla G(\mathbf{x})]$, where $\nabla G(\mathbf{x})=$ $\left(\partial G / \partial x_{1}, \ldots, \partial G / \partial x_{n}\right)$. If $d$ is not divisible by $p$, it can be extended to the whole of $\mathbb{P}_{K}^{n-1}$, since if $\nabla G(\mathbf{x})=\mathbf{0}$ then $d G(\mathbf{x})=\mathbf{x} \cdot \nabla G(\mathbf{x})=0$, so $G(\mathbf{x})=0$. Thus $\mathcal{G}$ is well-defined outside $V$.

REMARK 2.1. It is easy to prove that the fibres of $\mathcal{G}$ are finite. In particular, this implies that the polynomial $G^{\mathbf{y}}$, as defined above, cannot vanish identically for $\mathbf{y} \neq \mathbf{0}$, since then the image of $\mathbb{P}_{K}^{n-1}$ under the Gauss map would be contained in a hyperplane.

Lemma 2.1. Let $X \subseteq \mathbb{P}_{K}^{n-1}$ be an equidimensional subvariety of dimension $m$. Let $H \subset \mathbb{P}_{K}^{n-1}$ be a hypersurface such that $X \cap H$ is equidimensional of dimension $m-1$. Then

$$
(\operatorname{Sing} X) \cap H \subseteq \operatorname{Sing}(X \cap H) .
$$

In particular,

$$
\operatorname{dim} \operatorname{Sing}(X \cap H) \geq \operatorname{dim} \operatorname{Sing} X-1 .
$$

This is a standard result, and we omit the proof.

Notation 2.2. Let $F, G \in K\left[x_{1}, \ldots, x_{n}\right]$ be homogeneous polynomials, with $\operatorname{deg} G \geq 2$. For each $s=-1,0, \ldots, n-1$, define $T_{s}(F, G)$ to be the Zariski closed subset of $z=[\mathbf{z}] \in \mathbb{P}_{K}^{n-1}$ such that

$$
\operatorname{dim} \operatorname{Sing} \operatorname{Diff}_{\mathbf{z}}(F, G) \geq s .
$$

We define $T_{\operatorname{deg}}(F, G)$ to be the closed subset of $z \in \mathbb{P}_{K}^{n-1}$ such that

$$
\operatorname{dim} \operatorname{Diff}_{\mathbf{z}}(F, G)=\operatorname{dim} V(F, G) .
$$

We are interested in upper bounds for the dimension of $T_{s}(F, G)$. The version of Bertini's theorem that we shall use holds only in characteristic zero, whence the hypothesis in Lemma 2.2 .

Lemma 2.2. Suppose that $p=\operatorname{char} K=0$. Let $F, G \in K\left[x_{1}, \ldots, x_{n}\right]$ be homogeneous polynomials, with $\operatorname{deg} G \geq 2$. Suppose that $Y=V(F, G)$ is a non-singular complete intersection of dimension $n-3$. Suppose furthermore that $V(G)$ is non-singular. Then, for $-1 \leq s \leq n-3$, we have

$$
\operatorname{dim} T_{s}(F, G) \leq n-2-s .
$$

(If $s>n-3$, then of course $T_{s}(F, G)=\emptyset$.) 
Proof. Since $V(G)$ is non-singular, we can define the Gauss morphism

$$
\mathcal{G}: \mathbb{P}_{K}^{n-1} \rightarrow \mathbb{P}_{K}^{n-1}, \quad \mathbf{x} \mapsto\left(\xi_{1}, \ldots, \xi_{n}\right)=\left(\frac{\partial G}{\partial x_{1}}, \ldots, \frac{\partial G}{\partial x_{n}}\right) .
$$

Note that, using the notation $H_{\mathbf{z}}$ for the hyperplane $\mathbf{z} \cdot \boldsymbol{\xi}=0$, we have $\operatorname{Diff}_{\mathbf{z}}(F, G)=Y \cap \mathcal{G}^{-1}\left(H_{\mathbf{z}}\right)$. We shall recursively find a sequence of linear subspaces $\Pi_{-1}, \Pi_{0}, \ldots, \Pi_{n-3}$ of $\mathbb{P}_{K}^{n-1}$ such that $Y \cap \mathcal{G}^{-1}\left(\Pi_{s}\right)$ is non-singular of dimension $n-4-s$ for $s=-1,0, \ldots, n-3$. Let $\Pi_{-1}=\mathbb{P}_{K}^{n-1}$. Then $Y \cap \mathcal{G}^{-1}\left(\Pi_{-1}\right)=Y$ is non-singular by assumption. Suppose next that we have already found a linear subspace $\Pi_{s}, s \in\{-1,0, \ldots, n-4\}$ such that $Y_{s}:=Y \cap \mathcal{G}^{-1}\left(\Pi_{s}\right)$ is non-singular of dimension $n-4-s$, and let $\mathcal{G}_{s}: Y_{s} \rightarrow \Pi_{s}$ be the restriction of $\mathcal{G}$ to $Y_{s}$. Then, by Bertini's theorem [15, Cor. 6.11(2)], we may find a hyperplane $\Pi_{s+1} \subset \Pi_{s}$ such that $\mathcal{G}_{s}^{-1}\left(\Pi_{s+1}\right)=Y \cap \mathcal{G}^{-1}\left(\Pi_{s+1}\right)$ is non-singular of dimension $n-5-s$. Here we use the fact that $K$ has characteristic zero.

Now, for each $s=-1,0, \ldots, n-3$, let $\Lambda_{s}$ be the $s$-dimensional linear subspace of $\mathbb{P}_{K}^{n-1}=\operatorname{Proj} K\left[z_{1}, \ldots, z_{n}\right]$ parameterizing hyperplanes $H_{\mathbf{z}}$ such that $H_{\mathbf{z}} \supseteq \Pi_{s}$. We shall now prove that $T_{s}(F, G) \cap \Lambda_{s}=\emptyset$, and the statement will then follow from the projective dimension theorem. Therefore, suppose that $z=[\mathbf{z}] \in \Lambda_{s}$. Since in this case $H_{\mathbf{z}} \supseteq \Pi_{s}$, there is a linear subvariety $\Gamma_{z} \subseteq \mathbb{P}_{K}^{n-1}$ of codimension $s$ such that $\Pi_{s}=H_{\mathbf{z}} \cap \Gamma_{z}$. By the above, however,

$$
Y \cap \mathcal{G}^{-1}\left(H_{\mathbf{z}}\right) \cap \mathcal{G}^{-1}\left(\Gamma_{z}\right)=Y \cap \mathcal{G}^{-1}\left(\Pi_{s}\right)
$$

is non-singular, so by Lemma 2.1 we must have

$$
\left(\operatorname{Sing} \operatorname{Diff}_{\mathbf{z}}(F, G)\right) \cap \mathcal{G}^{-1}\left(\Gamma_{z}\right)=\emptyset .
$$

By Remark 2.1 it follows that

$$
\operatorname{dim} \mathcal{G}^{-1}\left(\Gamma_{z}\right)=\operatorname{dim} \Gamma_{z}=n-1-s .
$$

Now (4), along with the projective dimension theorem, implies that

$$
\operatorname{dim} \operatorname{Sing} \operatorname{Diff}_{\mathbf{z}}(F, G) \leq s-1 .
$$

Thus we have $z \notin T_{s}(F, G)$, as promised.

For the dimension of $T_{\operatorname{deg}}(F, G)$, we have the following result.

Lemma 2.3. Let $F, G \in K\left[x_{1}, \ldots, x_{n}\right]$ be homogeneous polynomials, with $p \nmid \operatorname{deg} G \geq 2$.

(i) Suppose that $Y=V(F, G)$ is a complete intersection of dimension $n-3$. Then

$$
\operatorname{dim} T_{\operatorname{deg}}(F, G) \leq 1 \text {. }
$$

(ii) Suppose furthermore that $n \geq 4$, and that both $Y$ and $V(G)$ are non-singular. Then $T_{\operatorname{deg}}(F, G)=\emptyset$. 
Proof. (i) As in the proof of Lemma 2.2, we have

$$
\operatorname{Diff}_{\mathbf{z}}(F, G)=Y \cap \mathcal{G}^{-1}\left(H_{\mathbf{z}}\right) .
$$

Thus, $z \in T_{\operatorname{deg}}(F, G)$ if and only if $W \subseteq \mathcal{G}^{-1}\left(H_{\mathbf{z}}\right)$ for some irreducible component $W$ of $Y$. This means that $\mathcal{G}(W) \subseteq H_{\mathbf{z}}$ for every $z \in T_{\operatorname{deg}}(F, G)$. By Remark 2.1 we have $\operatorname{dim} \mathcal{G}(W)=\operatorname{dim} W=n-3$, so there is a linear subspace $L \subset \mathbb{P}_{K}^{n-1}$ of dimension at least $n-3$ such that $L \subseteq H_{\mathbf{z}}$ for all $z \in T_{\operatorname{deg}}(F, G)$. In other words,

$$
T_{\mathrm{deg}}(F, G) \subseteq \Sigma(L):=\{H \in \mathbb{G}(n-2, n-1) ; L \subseteq H\} .
$$

We conclude that $\operatorname{dim} T_{\operatorname{deg}}(F, G) \leq \operatorname{dim} \Sigma(L) \leq 1$, proving (i).

(ii) Since $V(G)$ is non-singular, $G^{\mathbf{z}}$ does not vanish identically for $\mathbf{z} \neq \mathbf{0}$ by Remark 2.1. Thus it has $\operatorname{degree} \operatorname{deg} G-1$. Moreover, since $Y$ is a nonsingular complete intersection of dimension at least 1 , it is geometrically integral. Let $Y_{\mathbf{z}}=\operatorname{Diff}_{\mathbf{z}}(F, G)$.

Suppose now that $\operatorname{dim} Y_{\mathbf{z}}=\operatorname{dim} Y$. If $\bar{K}$ is an algebraic closure of $K$, then we would also have $\operatorname{dim}\left(Y_{\mathbf{z}}\right)_{\bar{K}}=\operatorname{dim} Y_{\bar{K}}$. Since $Y_{\bar{K}}$ is irreducible, this means that $V\left(G^{\mathbf{z}}\right) \subseteq Y$, implying, by the homogeneous Nullstellensatz, that $G^{\mathbf{z}} \in \operatorname{Rad}(F, G)$. However, the ideal $(F, G) \subset \bar{K}\left[x_{1}, \ldots, x_{n}\right]$ is prime, hence radical, so we would have $G^{\mathbf{z}} \in(F, G)$, which is impossible for degree reasons. This proves that $T_{\operatorname{deg}}(F, G)=\emptyset$.

We shall now extend Lemma 2.2 to the case of singular varieties. To this end, we shall use Bertini's theorem, in the following form.

Lemma 2.4. Suppose that $K$ is infinite. Let $X \subset \mathbb{P}_{K}^{n-1}$ be a complete intersection of degree $d$ and dimension $m . P u t \sigma=\operatorname{dim} \operatorname{Sing} X$. Then there exists a linear subspace $L \subseteq \mathbb{P}_{K}^{n-1}$ of codimension $\sigma+1$, such that $X \cap L$ is non-singular, of degree $d$ and dimension $m-\sigma-1$.

Proof. By Bertini's theorem [15, Cor. 6.11], there exists a hyperplane $\Gamma \subset \mathbb{P}_{K}^{n-1}$ such that

(i) $\Gamma$ intersects each irreducible component of $X$ properly,

(ii) $\Gamma$ intersects each irreducible component of Sing $X$ properly,

(iii) $(\operatorname{Reg} X) \cap \Gamma$ is non-singular.

Repeating this process, we get the desired result.

In fact, one can show that " $K$ is infinite" may be replaced by " $K$ has cardinality greater than some constant depending only on $n$ and $d "$ ". In the finite field case, one could then use the effective Bertini theorem proved by Ballico [1].

Lemma 2.5. Let $F, G \in K\left[x_{1}, \ldots, x_{n}\right]$ be homogeneous polynomials with $\operatorname{deg} G \geq 2$. Suppose that $Y=V(F, G)$ is a complete intersection of dimen- 
sion $n-3$. Let $\tilde{Y}=V(G)$ and define

$$
\sigma=\max \{\operatorname{dim} \operatorname{Sing} Y, \operatorname{dim} \operatorname{Sing} \tilde{Y}\} .
$$

(i) Suppose that $p=0$. Then, for $-1 \leq s \leq n-3$, we have

$$
\operatorname{dim} T_{\sigma+s+1}(F, G) \leq n-2-s .
$$

(ii) Suppose that $n \geq 5$ and $p \nmid \operatorname{deg} G$. Then

$$
\operatorname{dim} T_{\operatorname{deg}}(F, G) \leq \min \{\sigma, 1\} .
$$

Proof. (i) In case $\sigma=-1$, the statement follows directly from Lemma 2.2. so we assume that $\sigma \geq 0$. By Lemma 2.4 we can find a linear subspace $L \subset \mathbb{P}_{K}^{n-1}$ of codimension $\sigma+1$ such that $Y \cap L$ and $\tilde{Y} \cap L$ are non-singular. $L$ can be chosen in such a way that the degrees of the varieties are preserved and the dimensions decrease by $\sigma+1$.

Without loss of generality, assume that $L$ is given by $x_{n}=x_{n-1}=$ $\cdots=x_{n-\sigma}=0$. Then $Y_{0}=Y \cap L$ and $\tilde{Y}_{0}=\tilde{Y} \cap L$ are non-singular subschemes of $\mathbb{P}_{K}^{n-\sigma-2}=\operatorname{Proj} K\left[x_{1}, \ldots, x_{n-\sigma-1}\right]$. We have $Y_{0}=V\left(F_{0}, G_{0}\right)$ and $\tilde{Y}_{0}=V\left(G_{0}\right)$, where

$$
\begin{aligned}
& F_{0}\left(x_{1}, \ldots, x_{n-\sigma-1}\right)=F\left(x_{1}, \ldots, x_{n-\sigma-1}, 0, \ldots, 0\right), \\
& G_{0}\left(x_{1}, \ldots, x_{n-\sigma-1}\right)=G\left(x_{1}, \ldots, x_{n-\sigma-1}, 0, \ldots, 0\right) .
\end{aligned}
$$

For every $\mathbf{z}=\left(z_{1}, \ldots, z_{n-\sigma-1}, 0, \ldots, 0\right) \in L$, we have $\operatorname{Diff}_{\mathbf{z}}(F, G) \cap L=$ $\operatorname{Diff}_{\mathbf{z}_{0}}\left(F_{0}, G_{0}\right)$, where $\mathbf{z}_{0}=\left(z_{1}, \ldots, z_{n-\sigma-1}\right)$.

By repeated application of Lemma 2.1, we have

$$
T_{\sigma+s+1}(F, G) \cap L \subseteq T_{s}\left(F_{0}, G_{0}\right),
$$

and by Lemma 2.2 we have

$$
\operatorname{dim} T_{s}\left(F_{0}, G_{0}\right) \leq n-(\sigma+1)-2-s .
$$

Hence $\operatorname{dim} T_{\sigma+s+1}(F, G) \leq n-2-s$ by the projective dimension theorem.

(ii) In case $\sigma \neq 0$, the statement follows directly from Lemma 2.3. Thus, suppose that $\sigma=0$. Since $\operatorname{dim} T_{\operatorname{deg}}(F, G)=\operatorname{dim} T_{\operatorname{deg}}(F, G) \otimes \bar{K}$, we may assume that $K$ is infinite, and apply the construction above with a hyperplane $L \subset \mathbb{P}_{K}^{n-1}$. One easily sees that

$$
T_{\operatorname{deg}}(F, G) \cap L \subseteq T_{\operatorname{deg}}\left(F_{0}, G_{0}\right)=\emptyset,
$$

which implies that $\operatorname{dim} T_{\operatorname{deg}}(F, G) \leq 0$.

We shall apply the results above in the case when $G=F^{\mathbf{y}}$. Thus, we introduce the following notation.

Notation 2.3. If $V=V(F)$ is a hypersurface of degree at least 3 in $\mathbb{P}_{K}^{n-1}$, define

$$
V_{\mathbf{y}}=V\left(F, F^{\mathbf{y}}\right), \quad \tilde{V}_{\mathbf{y}}=V\left(F^{\mathbf{y}}\right),
$$


for any $\mathbf{y} \in K^{n}$, and let

$$
\begin{gathered}
s_{\mathbf{y}}(V)=\operatorname{dim} \operatorname{Sing} V_{\mathbf{y}}, \quad \tilde{s}_{\mathbf{y}}(V)=\operatorname{dim} \operatorname{Sing} \tilde{V}_{\mathbf{y}}, \\
\sigma_{\mathbf{y}}(V)=\max \left\{s_{\mathbf{y}}(V), \tilde{s}_{\mathbf{y}}(V)\right\} .
\end{gathered}
$$

Define $T_{\sigma}(V)$, for any $-1 \leq \sigma \leq n-1$, as the closed subset of $y \in \mathbb{P}_{K}^{n-1}$ such that $\sigma_{\mathbf{y}}(V) \geq \sigma$.

For any pair $(\mathbf{y}, \mathbf{z}) \in K^{n} \times K^{n}$, we define

$$
V_{\mathbf{y}, \mathbf{z}}=\operatorname{Diff}_{\mathbf{z}}\left(F, F^{\mathbf{y}}\right)=V\left(F, F^{\mathbf{y}}, F^{\mathbf{y}, \mathbf{z}}\right) .
$$

Furthermore, let $T_{\operatorname{deg}, \mathbf{y}}(V)=T_{\operatorname{deg}}\left(F, F^{\mathbf{y}}\right)$, and $T_{s, \mathbf{y}}(V)=T_{s}\left(F, F^{\mathbf{y}}\right)$ for $-1 \leq s \leq n-1$.

Lemma 2.6. Let $V$ be a non-singular hypersurface of degree $d \geq 3$ in $\mathbb{P}_{K}^{n-1}$. Then

$$
\operatorname{dim} V_{\mathbf{y}}=n-3
$$

for any $\mathbf{y} \in K^{n} \backslash\{\mathbf{0}\}$.

Proof. Let $F \in K\left[x_{1}, \ldots, x_{n}\right]$ be a generator for the ideal of $V$. Since $V$ is non-singular, $F^{\mathbf{y}}$ does not vanish identically by Remark 2.1, and thus has degree $d-1$. Moreover, since $V$ is non-singular of dimension at least 1 , it is geometrically integral.

Suppose now that $\operatorname{dim} V_{\mathbf{y}}=n-2$. If $\bar{K}$ is an algebraic closure of $K$, then we would also have $\operatorname{dim}\left(V_{\mathbf{y}}\right)_{\bar{K}}=n-2$. Since $V_{\bar{K}}$ is irreducible, this means that $V_{\bar{K}} \subseteq\left(V_{\mathbf{y}}\right)_{\bar{K}}$, implying, by the homogeneous Nullstellensatz, that $F^{\mathbf{y}} \in \operatorname{Rad}_{\bar{K}}(F)=(F)$. This is impossible for degree reasons. Thus $\operatorname{dim} V_{\mathbf{y}}=n-3$.

Applying Lemmata 2.3 and 2.5 in this case we get the following result.

Lemma 2.7. Let $V$ be a non-singular hypersurface of degree $d \geq 3$ in $\mathbb{P}_{K}^{n-1}$. Let $\mathbf{y} \in K^{n} \backslash\{\mathbf{0}\}$ and put $\sigma_{\mathbf{y}}=\sigma_{\mathbf{y}}(V)$.

(i) Suppose that $p=0$. Then, for $-1 \leq s \leq n-1$, we have

$$
\operatorname{dim} T_{\sigma_{\mathbf{y}}+s+1, \mathbf{y}}(V) \leq n-2-s .
$$

(ii) Suppose that $n \geq 5$ and $p \nmid(d-1)$. Then

$$
\operatorname{dim} T_{\operatorname{deg}, \mathbf{y}}(V) \leq \min \left\{\sigma_{\mathbf{y}}, 1\right\} .
$$

Proof. By Lemma 2.6 we have $\operatorname{dim} V_{\mathbf{y}}=n-3$. Now (i) is trivially true for $s \geq n-2$, and otherwise follows from Lemma 2.5. Part (ii) follows from Lemma 2.3 .

2.2. Results for polynomials over $\mathbb{Z}$. We have proved part (i) of Lemma 2.7 only in characteristic 0 . The aim of this section is to show that it is also true in characteristic $p$ for large enough $p$. Assume throughout this section that $n \geq 3$. 
Notation 2.4. If $f: X \rightarrow$ Spec $\mathbb{Z}$ is a morphism of schemes, we denote $f^{-1}((0))$ by $X_{0}$, and $f^{-1}((p))$ by $X_{p}$ for a prime $p \in \mathbb{Z}$.

Definition 2.2. Suppose that $F \in \mathbb{Z}\left[x_{1}, \ldots, x_{n}\right]$ is a homogeneous polynomial of degree $d \geq 3$. Let $p$ be a prime number or $p=0$. Let $Z=\operatorname{Proj} \mathbb{Z}\left[x_{1}, \ldots, x_{n}\right] /(F)$. Recall Notation 2.3 . We consider the following properties of $F$ (or $Z$ ):

$\left(\mathrm{R}_{0}(p)\right) Z_{p}$ is a non-singular variety.

$\left(\mathrm{R}_{1}(p)\right)$ For every $s=-1,0, \ldots, n-1$,

$$
\operatorname{dim} T_{s}\left(Z_{p}\right) \leq n-2-s .
$$

$\left(\mathrm{R}_{2}(p)\right)$ For every $\mathbf{y} \in \mathbb{F}_{p}^{n}$ and every $s=-1,0, \ldots, n-1$,

$$
\operatorname{dim} T_{\sigma_{\mathbf{y}}\left(Z_{p}\right)+s+1, \mathbf{y}}\left(Z_{p}\right) \leq n-2-s .
$$

In Section 2.1 it was shown that $\left(\mathrm{R}_{0}(0)\right)$ implies $\left(\mathrm{R}_{2}(0)\right)$. Combining the geometric results in [20] with [12, Lemma 2] one sees that if $F$ satisfies $\left(\mathrm{R}_{0}(0)\right)$, then $F$ satisfies $\left(\mathrm{R}_{0}(p)\right)$ and $\left(\mathrm{R}_{1}(p)\right)$ as soon as $p$ is large enough. Our aim in this section is to show the corresponding result for $\left(\mathrm{R}_{2}(p)\right)$.

Notation 2.5. Let $\mathcal{H}$ be the Hilbert scheme parameterizing degree $d$ hypersurfaces in $\mathbb{P}_{\mathbb{Z}}^{n-1}$. Then $\mathcal{H}$ can be identified with $\mathbb{P}_{\mathbb{Z}}^{D}$, where $D=$ $\left(\begin{array}{c}n-1+d \\ d\end{array}\right)-1$, and homogeneous coordinates for $\mathcal{H}$ are given by $\mathbf{t}=\left(t_{I}\right)$, where $I$ runs over all $n$-tuples $\left(i_{1}, \ldots, i_{n}\right)$ of non-negative integers such that $i_{1}+\cdots+i_{n}=d$. If $\mathbf{x}=\left(x_{1}, \ldots, x_{n}\right)$ are homogeneous coordinates for $\mathbb{P}_{\mathbb{Z}}^{n-1}$, then $\mathbf{x}^{I}$ denotes the monomial $x_{1}^{i_{1}} \cdots x_{n}^{i_{n}}$.

Furthermore, let

$$
\mathcal{P}=\mathcal{H} \times \mathbb{P}_{\mathbb{Z}}^{n-1} \times \mathbb{P}_{\mathbb{Z}}^{n-1} \times \mathbb{P}_{\mathbb{Z}}^{n-1} .
$$

Notation 2.6. Introduce multihomogeneous coordinates $(\mathbf{a}, \mathbf{y}, \mathbf{z}, \mathbf{x})$ on $\mathcal{P}$. Consider the following multihomogeneous polynomials:

$$
\begin{aligned}
& F(\mathbf{a}, \mathbf{y}, \mathbf{z}, \mathbf{x})=\sum a_{I} \mathbf{x}^{I}, \quad G(\mathbf{a}, \mathbf{y}, \mathbf{z}, \mathbf{x})=\sum y_{i} \frac{\partial F}{\partial x_{i}}, \\
& H(\mathbf{a}, \mathbf{y}, \mathbf{z}, \mathbf{x})=\sum z_{j} \frac{\partial G}{\partial x_{j}}=\sum_{i, j} y_{i} \frac{\partial^{2} F}{\partial x_{i} \partial x_{j}} z_{j} .
\end{aligned}
$$

(i) Let $\mathcal{M}$ be the closed subscheme of $\mathcal{P}$ defined by $F, G, H$ and all $3 \times 3$-minors of the matrix

$$
\left[\begin{array}{lll}
\partial F / \partial x_{1} & \cdots & \partial F / \partial x_{n} \\
\partial G / \partial x_{1} & \cdots & \partial G / \partial x_{n} \\
\partial H / \partial x_{1} & \cdots & \partial H / \partial x_{n}
\end{array}\right],
$$

and let $\pi_{\mathcal{M}}: \mathcal{M} \rightarrow \mathcal{H} \times \mathbb{P}_{\mathbb{Z}}^{n-1} \times \mathbb{P}_{\mathbb{Z}}^{n-1}$ be the projection onto the first three factors. 
(ii) Let $\mathcal{N}$ be the closed subscheme of $\mathcal{P}$ defined by $F, G$ and all $2 \times 2$ minors of the matrix

$$
\left[\begin{array}{lll}
\partial F / \partial x_{1} & \cdots & \partial F / \partial x_{n} \\
\partial G / \partial x_{1} & \cdots & \partial G / \partial x_{n}
\end{array}\right],
$$

and let $\pi_{\mathcal{N}}: \mathcal{N} \rightarrow \mathcal{H} \times \mathbb{P}_{\mathbb{Z}}^{n-1}$ be the projection onto the first two factors.

(iii) Let $\tilde{\mathcal{N}}$ be the closed subscheme of $\mathcal{P}$ defined by $G$ and its partial derivatives $\partial G / \partial x_{1}, \ldots, \partial G / \partial x_{n}$, and let $\pi_{\tilde{\mathcal{N}}}: \tilde{\mathcal{N}} \rightarrow \mathcal{H} \times \mathbb{P}_{\mathbb{Z}}^{n-1}$ be the projection onto the first two factors.

Notation 2.7. Suppose that $a \in \mathcal{H}$ and write $k=k(a)$. Suppose that $y, z \in \mathbb{P}_{k}^{n-1}$. Then we define

$$
\begin{gathered}
\mathbf{S}(a, y, z)=\pi_{\mathcal{M}}^{-1}((a, y, z)), \quad \mathbf{S}(a, y)=\pi_{\mathcal{N}}^{-1}((a, y)), \quad \tilde{\mathbf{S}}(a, y)=\pi_{\tilde{\mathcal{N}}}^{-1}((a, y)), \\
\sigma(a, y)=\max \{\operatorname{dim} \mathbf{S}(a, y), \operatorname{dim} \tilde{\mathbf{S}}(a, y)\} .
\end{gathered}
$$

Also, for each $s \in\{-1,0,1, \ldots, n-1\}$, define

$$
\mathbf{T}_{s}(a, y)=\left\{z \in \mathbb{P}_{k}^{n-1} ; \operatorname{dim} \mathbf{S}(a, y, z) \geq s\right\} .
$$

$\mathbf{T}_{s}(a, y)$ is a closed subset of $\mathbb{P}_{k}^{n-1}$, by Chevalley's theorem on upper semicontinuity of fibre dimension [6, Cor. 13.1.5]. Let us relate Notation 2.7 to Notation 2.3. If $k$ is a perfect field, and $V$ is the hypersurface of $\mathbb{P}_{k}^{n-1}$ corresponding to $a$, then the Jacobian criterion [18, $\$ 4.2]$ implies that $\mathbf{S}(a, y, z)=$ $\operatorname{Sing} V_{\mathbf{y}, \mathbf{z}}, \mathbf{S}(a, y)=\operatorname{Sing} V_{\mathbf{y}}$ and $\tilde{\mathbf{S}}(a, y)=\operatorname{Sing} \tilde{V}_{\mathbf{y}}$. Thus, in this case, $\mathbf{T}_{s}(a, y)=T_{s, \mathbf{y}}(V)$.

Notation 2.8. Let $\mathcal{R}_{2}$ be the set of $a \in \mathcal{H}$ such that for all $y \in \mathbb{P}_{k(a)}^{n-1}$ and all $s$, we have $\operatorname{dim}\left(\mathbf{T}_{\sigma(a, y)+s+1}(a, y)\right) \leq n-2-s$.

REMARK 2.2. If $\mathbf{a} \in \mathbb{Z}^{D+1}$ is the tuple of coefficients of $F \in \mathbb{Z}\left[x_{1}, \ldots, x_{n}\right]$, then $F$ satisfies $\left(\mathrm{R}_{2}(p)\right)$ if and only if $\mathbf{a}(\bmod p)$ belongs to $\mathcal{R}_{2}\left(\mathbb{F}_{p}\right)$.

Recall that a subset of a Noetherian topological space $X$ is constructible if it can be written as a finite union of locally closed subsets of $X$ [4, 0, 9.1.7].

Our key argument in deriving a criterion for $\left(\mathrm{R}_{2}(p)\right)$ is the following fact, the proof of which uses a version of "quantifier elimination" for schemes, developed by Chevalley and Grothendieck.

Lemma 2.8. $\mathcal{R}_{2}$ is a constructible subset of $\mathcal{H}$.

Proof. Let $\mathcal{U}_{r}$, for $r \in\{-1,0,1, \ldots, n-1\}$, be the set of points $(a, y)$ $\in \mathcal{H} \times \mathbb{P}_{\mathbb{Z}}^{n-1}$ such that $\sigma(a, y) \leq r$. Furthermore, for each pair $(s, u)$ $\in\{-1,0,1, \ldots, n-1\}^{2}$, let $\mathcal{Q}_{s, u}$ be the set of points $(a, y) \in \mathcal{H} \times \mathbb{P}_{\mathbb{Z}}^{n-1}$ such that $\operatorname{dim}\left(\mathbf{T}_{s}(a, y)\right)>u$. Using the semicontinuity theorem again, one sees that $\mathcal{U}_{r}$ is open and $\mathcal{Q}_{s, u}$ is closed. 
Thus, the set

$$
\mathcal{S}:=\bigcup_{-1 \leq s, \sigma \leq n-1} \mathcal{U}_{\sigma} \cap \mathcal{Q}_{\sigma+s+1, n-2-s}
$$

is a constructible subset of $\mathcal{H} \times \mathbb{P}_{\mathbb{Z}}^{n-1}$. If $\pi: \mathcal{H} \times \mathbb{P}_{\mathbb{Z}}^{n-1} \rightarrow \mathcal{H}$ denotes the projection onto the first factor, then by [5, IV, $1.8 .4], \pi(\mathcal{S})$ is a constructible subset of $\mathcal{H}$. Since $\mathcal{R}_{2}=\mathcal{H} \backslash \pi(\mathcal{S})$, and the family of constructible subsets is closed under complements, we have proved the lemma.

As a consequence, we get the following result, which motivates this section. $\|F\|$ denotes the maximum of the absolute values of the coefficients of $F$.

Corollary 2.1. For each homogeneous polynomial $F \in \mathbb{Z}\left[x_{1}, \ldots, x_{n}\right]$ of degree $d \geq 3$ defining a non-singular hypersurface in $\mathbb{P}_{\mathbb{Q}}^{n-1}$ (i.e. satisfying $\left.\left(\mathrm{R}_{0}(0)\right)\right)$, the set of primes $\mathcal{P}(F)$ such that $F$ does not satisfy all of the conditions $\left(\mathrm{R}_{0}(p)\right),\left(\mathrm{R}_{1}(p)\right)$ and $\left(\mathrm{R}_{2}(p)\right)$ is finite. Furthermore, there are constants $C, \kappa$, depending only on $n$ and $d$, such that

$$
\prod_{p \in \mathcal{P}(F)} p \leq C\|F\|^{\kappa} .
$$

Proof. Let $\mathcal{P}_{2}(F)$ be the set of primes $p$ for which $F$ does not satisfy $\left(\mathrm{R}_{2}(p)\right)$. Taking into account the results mentioned after Definition 2.2 , it suffices to prove that $\mathcal{P}_{2}(F)$ is finite and that

$$
\prod_{p \in \mathcal{P}_{2}(F)} p \leq C\|F\|^{\kappa}
$$

for constants $C, \kappa=O_{n, d}(1)$.

By Lemma 2.8, we can write

$$
\mathcal{R}_{2}=\bigcup_{i=1}^{k} A_{i} \cap S_{i},
$$

where the $A_{i}$ are open and the $S_{i}$ are closed. We may assume that $A_{i}=$ $D_{+}\left(f_{i}\right)$ for homogeneous polynomials $f_{i} \in \mathbb{Z}\left[x_{1}, \ldots, x_{n}\right]$ (see [11, §II.2]).

Suppose now that $F$ satisfies the hypotheses of the corollary, and let $\mathbf{a} \in \mathbb{Z}^{D+1}$ be the tuple of coefficients of $F$. Then $\mathbf{a} \in \mathcal{R}_{2}(\mathbb{Q})$, so there is an index $i$ such that $\mathbf{a} \in S_{i}(\mathbb{Q})$ and $f_{i}(\mathbf{a}) \neq 0$. For every $p \in \mathcal{P}_{2}(F)$, we then have $p \mid f_{i}(\mathbf{a})$, since $\mathbf{a} \in S_{i}(\mathbb{Q})$ implies $\mathbf{a}(\bmod p) \in S_{i}\left(\mathbb{F}_{p}\right)$. Thus, $\mathcal{P}_{2}(F)$ is a finite set and

$$
\prod_{p \in \mathcal{P}_{2}(F)} p \leq\left|f_{i}(\mathbf{a})\right| \leq\left\|f_{j}\right\||\mathbf{a}|^{\kappa}
$$

where $\kappa=\operatorname{deg} f_{j}$. 
3. Preliminary number-theoretic results. We begin with some remarks on the results from the author's paper [19] that we will use.

REMARK 3.1. The error term

$$
D_{n+1} B^{s+1} q^{(n-r-s-2) / 2}\left(B+q^{1 / 2}\right)
$$

in [19, Theorem 3.3] can be given by the simpler expression

$$
D_{n+1} B^{s+2} q^{(n-r-s-2) / 2} \text {. }
$$

Indeed, if $q^{1 / 2} \gg B$, then one would have $B^{s+2} q^{(n-r-s-2) / 2} \gg B^{n-r}$, so that the theorem would be true by means of a trivial estimate, such as [19, Lemma 3.1].

We shall in the proof use the weighted asymptotic formula mentioned in [19, Remark 4.4]. Let us therefore state this result. Appealing to Remark 3.1. we may simplify the error term somewhat.

Theorem 3.1. Let $W: \mathbb{R}^{n} \rightarrow[0,1]$ be an infinitely differentiable function supported on $[-2,2]^{n}$. Let $f_{1}, \ldots, f_{r}$ be polynomials in $\mathbb{Z}\left[x_{1}, \ldots, x_{n}\right]$ of degree at least 3 , with leading forms $F_{1}, \ldots, F_{r}$. Let

$$
Z=\operatorname{Proj} \mathbb{Z}\left[x_{1}, \ldots, x_{n}\right] /\left(F_{1}, \ldots, F_{r}\right)
$$

and suppose that $p$ and $q$ are primes, with $p \leq B \leq q$, such that both $Z_{p}$ and $Z_{q}$ are non-singular subvarieties of $\mathbb{P}_{\mathbb{F}_{q}}^{n-1}$ of dimension $n-1-r$. Then

$$
\begin{aligned}
& N_{W}\left(f_{1}, \ldots, f_{r}, B, p q\right)-p^{-r} q^{-r} N_{W}(0, B, p q) \\
& \ll W, n, d, C \\
& B^{(n+1) / 2} p^{-r / 2} q^{(n-r-1) / 4}+B^{(n+1) / 2} p^{(n-2 r) / 2} q^{-1 / 4} \\
& +B^{n} p^{-(n+r-1) / 2} q^{-r}+B^{n-C / 2} p^{(C-r) / 2} q^{-r / 2}
\end{aligned}
$$

for any $C>0$, where $d=\max _{i}\left(\operatorname{deg} f_{i}\right)$.

The following result is standard [19, Lemma 3.1].

Proposition 3.1. Let $X=\operatorname{Spec} \mathbb{F}_{q}\left[x_{1}, \ldots, x_{n}\right] /\left(f_{1}, \ldots, f_{\rho}\right)$ be a closed subscheme of $\mathbb{A}_{\mathbb{F}_{q}}^{n}$, and let $d=\max _{i}\left(\operatorname{deg} f_{i}\right)$. For any box

$$
\mathrm{B}=\left[a_{1}-b_{1}, a_{1}+b_{1}\right] \times \cdots \times\left[a_{n}-b_{n}, a_{n}+b_{n}\right],
$$

with $\left|b_{i}\right| \leq B$, containing at most one representative of each congruence class modulo $q$, let $\mathrm{B}_{q}$ be its image in $(\mathbb{Z} / q \mathbb{Z})^{n}$. Then

$$
\#\left(\mathrm{~B}_{q} \cap X\left(\mathbb{F}_{q}\right)\right) \ll_{n, \rho, d} B^{\operatorname{dim} X} .
$$

REMARK. The dependence on $\rho$ can be eliminated - one can show [17, Cor. V.1.5] that there is an ideal generated by at most $n$ elements whose radical equals the radical of $\left(f_{1}, \ldots, f_{\rho}\right)$.

The following asymptotic formula for the number of rational points on a complete intersection, due to Hooley [14, is a consequence of the Weil conjectures [3]. The version below is proved in [19, Lemma 3.2]. 
Proposition 3.2. Let $f_{1}, \ldots, f_{r}$ be polynomials in $\mathbb{F}_{q}\left[x_{1}, \ldots, x_{n}\right]$ with leading forms $F_{1}, \ldots, F_{r}$, respectively. Let

$$
\begin{aligned}
X & =\operatorname{Spec} \mathbb{F}_{q}\left[x_{1}, \ldots, x_{n}\right] /\left(f_{1}, \ldots, f_{r}\right), \\
Z & =\operatorname{Proj} \mathbb{F}_{q}\left[x_{1}, \ldots, x_{n}\right] /\left(F_{1}, \ldots, F_{r}\right) .
\end{aligned}
$$

Suppose that $\operatorname{dim} Z=n-1-r$ and let $s=\operatorname{dim} \operatorname{Sing} Z$. Then

$$
\# X\left(\mathbb{F}_{q}\right)=q^{n-r}+O_{n, d}\left(q^{(n-r+2+s) / 2}\right),
$$

where $d=\max _{i}\left(\operatorname{deg} F_{i}\right)$.

The following result is a simple exercise in Poisson summation. The argument appears in [12].

Lemma 3.1. Let $\phi: \mathbb{R}^{n} \rightarrow \mathbb{R}$ be an infinitely differentiable function supported in the box $[-M, M]^{n}$, and let $D_{k}$, for $k=0,1, \ldots$, be the maximum over $\mathbb{R}^{n}$ of all partial derivatives of $\phi$ of order $k$. Let $a$ and $B$ be real numbers such that $B \geq 1$ and $1 \leq a \leq B$. Then

$$
\begin{aligned}
\sum_{\mathbf{x} \in \mathbb{Z}^{n}} \phi\left(\frac{1}{B} \mathbf{x}\right) \sum_{\mathbf{y} \in \mathbb{Z}^{n}} \phi\left(\frac{1}{B}(\mathbf{x}+a \mathbf{y})\right) \\
=a^{-n}\left(\sum_{\mathbf{x} \in \mathbb{Z}^{n}} \phi\left(\frac{1}{B} \mathbf{x}\right)\right)^{2}+O_{n, M, k}\left(D_{0} D_{k} B^{2 n-k} a^{-n+k}\right) \\
+O_{n, M, k}\left(D_{k}^{2} B^{2(n-k)} a^{-n+k}\right)
\end{aligned}
$$

for any $k \in \mathbb{Z}_{\geq 0}$.

Proof. Since $\phi$ is infinitely differentiable and compactly supported, we have for the Fourier transform $\hat{\phi}$ the estimate

$$
\hat{\phi}(\boldsymbol{\xi}) \ll_{n, M, k} D_{k}|\boldsymbol{\xi}|^{-k} .
$$

The function $\Phi(\mathbf{x})=\phi((1 / B) \mathbf{x})$ has the Fourier transform $\hat{\Phi}(\boldsymbol{\xi})=B^{n} \hat{\phi}(B \boldsymbol{\xi})$. Thus, by Poisson's summation formula and (6), we get

$$
\sum_{\mathbf{x} \in \mathbb{Z}^{n}} \phi\left(\frac{1}{B} \mathbf{x}\right)=B^{n} \sum_{\boldsymbol{\xi} \in \mathbb{Z}^{n}} \hat{\phi}(B \boldsymbol{\xi})=B^{n} \hat{\phi}(\mathbf{0})+O_{n, M, k}\left(D_{k} B^{n-k}\right) .
$$

For fixed $\mathbf{x}$, put $\psi(\mathbf{y})=\phi((1 / B)(\mathbf{x}+a \mathbf{y}))$. Then

$$
\hat{\psi}(\boldsymbol{\eta})=\left(\frac{B}{a}\right)^{n} \exp \left(-2 \pi i a^{-1} \mathbf{x} \cdot \boldsymbol{\eta}\right) \hat{\phi}\left(\frac{B}{a} \boldsymbol{\eta}\right) .
$$

By Poisson's summation formula and (7) we calculate 


$$
\begin{aligned}
\sum_{\mathbf{y} \in \mathbb{Z}^{n}} \phi\left(\frac{1}{B}(\mathbf{x}+a \mathbf{y})\right) & =\left(\frac{B}{a}\right)^{n} \sum_{\boldsymbol{\eta} \in \mathbb{Z}^{n}} \exp \left(-2 \pi i a^{-1} \mathbf{x} \cdot \boldsymbol{\eta}\right) \hat{\phi}\left(\frac{B}{a} \boldsymbol{\eta}\right) \\
& =a^{-n}\left(B^{n} \hat{\phi}(\mathbf{0})+O_{n, M, k}\left(D_{k} B^{n-k} a^{k}\right)\right) \\
& =a^{-n} \sum_{\mathbf{v} \in \mathbb{Z}^{n}} \phi\left(\frac{1}{B} \mathbf{v}\right)+O_{n, M, k}\left(D_{k} B^{n-k} a^{-n+k}\right)
\end{aligned}
$$

Multiplying (8) by $\phi\left(\frac{1}{B} \mathbf{x}\right)$, summing over $\mathbf{x} \in \mathbb{Z}^{n}$ and using (7) and (6) we get the desired formula.

4. The density of solutions to $f(\mathbf{x}) \equiv 0(\bmod \pi p q)$. This section constitutes the technical heart of the proof of Theorem 1.1. Let $n \geq 5$, and let $f$ be a polynomial in $\mathbb{Z}\left[x_{1}, \ldots, x_{n}\right]$ of degree $d \geq 4$, with leading form $F$. Let $W$ be the infinitely differentiable weight function in (3).

Notation 4.1 (Differenced polynomials). For any $\mathbf{y} \in \mathbb{Z}^{n}$, define the polynomial $f^{\mathbf{y}} \in \mathbb{Z}\left[x_{1}, \ldots, x_{n}\right]$ by

$$
f^{\mathbf{y}}(\mathbf{x})=f(\mathbf{x}+\mathbf{y})-f(\mathbf{x}) .
$$

For any pair $(\mathbf{y}, \mathbf{z}) \in \mathbb{Z}^{n} \times \mathbb{Z}^{n}$, define

$$
f^{\mathbf{y}, \mathbf{z}}(\mathbf{x})=f(\mathbf{x}+\mathbf{y}+\mathbf{z})-f(\mathbf{x}+\mathbf{y})-f(\mathbf{x}+\mathbf{z})+f(\mathbf{x}) .
$$

Furthermore, let

$$
\begin{gathered}
F^{\mathbf{y}}(\mathbf{x})=\mathbf{y} \cdot \nabla F(\mathbf{x})=y_{1} \frac{\partial F}{\partial x_{1}}+\cdots+y_{n} \frac{\partial F}{\partial x_{n}}, \\
F^{\mathbf{y}, \mathbf{z}}(\mathbf{x})=(\operatorname{Hess} F) \mathbf{y} \cdot \mathbf{z}=\sum_{1 \leq i, j \leq n} \frac{\partial^{2} F}{\partial x_{i} \partial x_{j}} y_{i} z_{j},
\end{gathered}
$$

as in Section 2

For any prime $p$, define the schemes

$$
\begin{gathered}
Z_{p, \mathbf{y}}=\operatorname{Proj} \mathbb{Z}\left[x_{1}, \ldots, x_{n}\right] /\left(p, F, F^{\mathbf{y}}\right), \quad \tilde{Z}_{p, \mathbf{y}}=\operatorname{Proj} \mathbb{Z}\left[x_{1}, \ldots, x_{n}\right] /\left(p, F^{\mathbf{y}}\right), \\
Z_{p, \mathbf{y}, \mathbf{z}}=\operatorname{Proj} \mathbb{Z}\left[x_{1}, \ldots, x_{n}\right] /\left(p, F, F^{\mathbf{y}}, F^{\mathbf{y}, \mathbf{z}}\right) .
\end{gathered}
$$

Put

$$
\begin{aligned}
& s_{p}(\mathbf{y})=\operatorname{dim} \operatorname{Sing} Z_{p, \mathbf{y}}, \quad \sigma_{p}(\mathbf{y})=\max \left\{s_{p}(\mathbf{y}), \tilde{s}_{p}(\mathbf{y})\right\}, \\
& \tilde{s}_{p}(\mathbf{y})=\operatorname{dim} \operatorname{Sing} \tilde{Z}_{p, \mathbf{y}}, \quad s_{p}(\mathbf{y}, \mathbf{z})=\operatorname{dim} \operatorname{Sing} Z_{p, \mathbf{y}, \mathbf{z}} .
\end{aligned}
$$

If $Z_{p}=\operatorname{Proj} \mathbb{Z}\left[x_{1}, \ldots, x_{n}\right] /(p, F)$, we have $s_{p}(\mathbf{y})=s_{\mathbf{y}_{p}}\left(Z_{p}\right), \tilde{s}_{p}(\mathbf{y})=\tilde{s}_{\mathbf{y}_{p}}\left(Z_{p}\right)$, $\sigma_{p}(\mathbf{y})=\sigma_{\mathbf{y}_{p}}\left(Z_{p}\right)$ (cf. Notation 2.3), where $\mathbf{y}_{p}$ denotes the image of $\mathbf{y}$ under the natural map $\mathbb{Z}^{n} \rightarrow(\mathbb{Z} / p \mathbb{Z})^{n}$.

Notation 4.2 ("Differenced" weight functions). For any $\mathbf{a} \in \mathbb{R}^{n}$, let the infinitely differentiable function $W_{\mathbf{a}}$ be given by

$$
W_{\mathbf{a}}(\mathbf{x})=W(\mathbf{x}) W(\mathbf{x}+\mathbf{a}) .
$$


Note that $W_{\mathbf{a}}$ vanishes identically if $|\mathbf{a}| \geq 4$. Also define, for any pair $\left(\mathbf{a}, \mathbf{a}^{\prime}\right) \in$ $\mathbb{R}^{n} \times \mathbb{R}^{n}$, the function

$$
W_{\mathbf{a}, \mathbf{a}^{\prime}}(\mathbf{x})=W_{\mathbf{a}}(\mathbf{x}) W_{\mathbf{a}}\left(\mathbf{x}+\mathbf{a}^{\prime}\right)=W(\mathbf{x}) W(\mathbf{x}+\mathbf{a}) W\left(\mathbf{x}+\mathbf{a}^{\prime}\right) W\left(\mathbf{x}+\mathbf{a}+\mathbf{a}^{\prime}\right) .
$$

Suppose that we are given three different prime numbers $\pi, p, q$, with $\pi, p \leq B<q / 4$, such that $F$ satisfies

$$
\begin{aligned}
& \left(\mathrm{R}_{0}(\pi)\right), \\
& \left(\mathrm{R}_{0}(p)\right),\left(\mathrm{R}_{1}(p)\right), \\
& \left(\mathrm{R}_{0}(q)\right),\left(\mathrm{R}_{1}(q)\right) \text { and }\left(\mathrm{R}_{2}(q)\right)
\end{aligned}
$$

(see Definition 2.2). We shall later prove the existence of suitable primes $\pi, p, q$.

LEMMA 4.1. Under the hypotheses above, we have the following results:

(i) Put

$$
K=\pi^{-n} p^{-1} q^{-1} N_{W}(0, B, \pi p q) .
$$

Then

$$
\begin{aligned}
N_{W}(f, B, \pi p q)= & (\pi p q)^{-1} N_{W}(0, B, \pi p q)+O\left(\pi^{(n-1) / 2} \Sigma^{1 / 2}\right) \\
& +O\left(B^{n} \pi^{-n / 2} p^{-1} q^{-1}\right),
\end{aligned}
$$

where

$$
\Sigma=\sum_{\mathbf{u} \in \mathbb{F}_{\pi}^{n}}\left(\sum_{\substack{\mathbf{x} \equiv \mathbf{u}(\pi) \\ p q \mid f(\mathbf{x})}} W\left(\frac{1}{B} \mathbf{x}\right)-K\right)^{2} .
$$

(ii) For any $\mathbf{y} \in \mathbb{Z}^{n}$, put

Then

$$
\Delta(\mathbf{y})=\sum_{\substack{\mathbf{x} \in \mathbb{Z}^{n} \\ p q|f(\mathbf{x}) \\ p q| f(\mathbf{x}+\pi \mathbf{y})}} W_{\pi \mathbf{y}}\left(\frac{1}{B} \mathbf{x}\right)-p^{-2} q^{-2} \sum_{\mathbf{x} \in \mathbb{Z}^{n}} W_{\pi \mathbf{y}}\left(\frac{1}{B} \mathbf{x}\right) .
$$

$$
\Sigma=\sum_{|\mathbf{y}| \leq 4 B / \pi} \Delta(\mathbf{y})+\varepsilon_{1}
$$

(iii) Suppose that $\mathbf{y} \neq 0$. For any $\mathbf{z} \in \mathbb{Z}^{n}$, put

$$
\Delta(\mathbf{y}, \mathbf{z}):=\sum_{\substack{\mathbf{x} \in \mathbb{Z}^{n} \\ q|f(\mathbf{x}) \\ q| f^{p \mathbf{z}}(\mathbf{x}) \\ q \mid f^{\pi \mathbf{y}, p \mathbf{z}}(\mathbf{x})}} W_{\pi \mathbf{y}, p \mathbf{z}}\left(\frac{1}{B} \mathbf{x}\right)-q^{-3} \sum_{\mathbf{x} \in \mathbb{Z}^{n}} W_{\pi \mathbf{y}, p \mathbf{z}}\left(\frac{1}{B} \mathbf{x}\right) .
$$

Then

$$
\Delta(\mathbf{y})=p^{(n-2) / 2}\left(\sum_{|\mathbf{z}| \leq 4 B / p} \Delta(\mathbf{y}, \mathbf{z})\right)^{1 / 2}+\varepsilon_{2}(\mathbf{y})+\varepsilon_{3} .
$$


(iv) Furthermore,

$$
\Delta(\mathbf{0}) \ll B^{n} p^{-1} q^{-1}+\varepsilon_{0} .
$$

All the implied constants depend only on $n$ and d, unless otherwise specified. The error terms $\mathcal{E}_{i}$ satisfy the following estimates:

$$
\begin{aligned}
\mathcal{E}_{0} \ll & B^{(n+1) / 2} p^{-1 / 2} q^{(n-2) / 4}+B^{(n+1) / 2} p^{(n-2) / 2} q^{-1 / 4}+B^{n} p^{-n / 2} q^{-1}, \\
\mathcal{E}_{1} \ll{ }_{C} & B^{(3 n+1) / 2} \pi^{-n} p^{-3 / 2} q^{(n-6) / 4}+B^{(3 n+1) / 2} \pi^{-n} p^{(n-4) / 2} q^{-5 / 4} \\
& \quad+B^{2 n} \pi^{-n} p^{-(n+2) / 2} q^{-2}+B^{2 n-C} \pi^{-n+C} p^{-2} q^{-2} \quad \text { for any } C>0,
\end{aligned}
$$

$\varepsilon_{2}(\mathbf{y}) \ll B^{n} p^{\left(n-s_{p}(\mathbf{y})\right) / 2} q^{-2}$,

$\mathcal{E}_{3} \ll_{C} B^{(n+1) / 2} p^{-1} q^{(n-6) / 4}+B^{n-C} p^{-1+C} q^{-3 / 2} \quad$ for any $C>0$.

Proof. Starting from the definition of $N_{W}(f, B, \pi p q)$, we write

$$
\begin{aligned}
N_{W}(f, B, \pi p q) & =\sum_{\substack{\mathbf{u} \in \mathbb{F}_{\pi}^{n} \\
f_{\pi}(\mathbf{u})=0}}\left(\sum_{\substack{\mathbf{x} \equiv \mathbf{u}(\pi) \\
p q \mid f(\mathbf{x})}} W\left(\frac{1}{B} \mathbf{x}\right)-K\right)+K \sum_{\substack{\mathbf{u} \in \mathbb{F}_{\pi}^{n} \\
f_{\pi}(\mathbf{u})=0}} 1 \\
& =S+K\left(\pi^{n-1}+O\left(\pi^{n / 2}\right)\right),
\end{aligned}
$$

where

$$
S=\sum_{\substack{\mathbf{u} \in \mathbb{F}^{n} \\ f_{\pi}(\mathbf{u})=0}}\left(\sum_{\substack{\mathbf{x} \equiv \mathbf{u}(\pi) \\ p q \mid f(\mathbf{x})}} W\left(\frac{1}{B} \mathbf{x}\right)-K\right),
$$

and $f_{\pi} \in \mathbb{Z} / \pi \mathbb{Z}\left[x_{1}, \ldots, x_{n}\right]$ is the image of $f$ under the natural homomorphism $\mathbb{Z}\left[x_{1}, \ldots, x_{n}\right] \rightarrow \mathbb{Z} / \pi \mathbb{Z}\left[x_{1}, \ldots, x_{n}\right]$. Here we have used the property $\left(\mathrm{R}_{0}(\pi)\right)$, applying Proposition 3.2 to the hypersurface defined by $f_{\pi}(\mathbf{u})=0$. By Cauchy's inequality,

$$
S^{2} \ll \pi^{n-1} \sum_{\mathbf{u} \in \mathbb{F}_{\pi}^{n}}\left(\sum_{\substack{\mathbf{x} \equiv \mathbf{u}(\pi) \\ p q \mid f(\mathbf{x})}} W\left(\frac{1}{B} \mathbf{x}\right)-K\right)^{2},
$$

so we have

$$
N_{W}(f, B, \pi p q)=\pi^{n-1} K+O\left(\pi^{(n-1) / 2} \Sigma^{1 / 2}\right)+O\left(B^{n} \pi^{-n / 2} p^{-1} q^{-1}\right),
$$

and (i) is proved.

Now,

$$
\begin{aligned}
\Sigma & =\sum_{\mathbf{u} \in \mathbb{F}_{\pi}^{n}}\left(\sum_{\substack{\mathbf{x} \equiv \mathbf{u}(\pi) \\
p q \mid f(\mathbf{x})}} W\left(\frac{1}{B} \mathbf{x}\right)-K\right)^{2} \\
& =\sum_{\mathbf{u} \in \mathbb{F}_{\pi}^{n}}\left(\sum_{\substack{\mathbf{x} \equiv \mathbf{u}(\pi) \\
p q \mid f(\mathbf{x})=0}} W\left(\frac{1}{B} \mathbf{x}\right)\right)^{2}-2 K N_{W}(f, B, p q)+\pi^{n} K^{2} .
\end{aligned}
$$


Using Theorem 3.1, along with the properties $\left(\mathrm{R}_{0}(p)\right)$ and $\left(\mathrm{R}_{0}(q)\right)$, we have

$$
N_{W}(f, B, p q)=\pi^{n} K+\varepsilon_{0},
$$

where

$$
\mathcal{E}_{0} \ll B^{(n+1) / 2} p^{-1 / 2} q^{(n-2) / 4}+B^{(n+1) / 2} p^{(n-2) / 2} q^{-1 / 4}+B^{n} p^{-n / 2} q^{-1} .
$$

(The last error term in Theorem 3.1 becomes negligible upon taking $C \geq$ $n-1$.) We conclude that

$$
\Sigma=\sum_{\mathbf{u} \in \mathbb{F}_{\pi}^{n}}\left(\sum_{\substack{\mathbf{x} \equiv \mathbf{u}(\pi) \\ p q \mid f(\mathbf{x})}} W\left(\frac{1}{B} \mathbf{x}\right)\right)^{2}-\pi^{n} K^{2}+K \mathcal{E}_{0} .
$$

Introducing a new variable $\mathbf{y}$, we expand the sum of squares as a double sum

$$
\begin{aligned}
\sum_{\mathbf{u} \in \mathbb{F}_{\pi}^{n}}\left(\sum_{\substack{\mathbf{x} \equiv \mathbf{u}(\pi) \\
p q \mid f(\mathbf{x})}} W\left(\frac{1}{B} \mathbf{x}\right)\right)^{2} & =\sum_{\substack{\mathbf{x} \in \mathbb{Z}^{n} \\
p q \mid f(\mathbf{x})}} W\left(\frac{1}{B} \mathbf{x}\right) \sum_{\substack{\mathbf{y} \in \mathbb{Z}^{n} \\
p q \mid f(\mathbf{x}+\pi \mathbf{y})}} W\left(\frac{1}{B}(\mathbf{x}+\pi \mathbf{y})\right) \\
& =\sum_{|\mathbf{y}| \leq 4 B / \pi} \sum_{\substack{\mathbf{x} \in \mathbb{Z}^{n} \\
p q|f(\mathbf{x}) \\
p q| f(\mathbf{x}+\pi \mathbf{y})}} W_{\pi \mathbf{y}}\left(\frac{1}{B} \mathbf{x}\right) .
\end{aligned}
$$

Recalling the definition of $\Delta(\mathbf{y})$ above, we have

$$
\Sigma=\sum_{|\mathbf{y}| \leq 4 B / \pi} \Delta(\mathbf{y})+p^{-2} q^{-2} \sum_{\mathbf{y} \in \mathbb{Z}^{n}} \sum_{\mathbf{x} \in \mathbb{Z}^{n}} W_{\pi \mathbf{y}}\left(\frac{1}{B} \mathbf{x}\right)-\pi^{n} K^{2}+K \varepsilon_{0} .
$$

By Lemma 3.1, however,

$$
p^{-2} q^{-2} \sum_{\mathbf{y} \in \mathbb{Z}^{n}} \sum_{\mathbf{x} \in \mathbb{Z}^{n}} W_{\pi \mathbf{y}}\left(\frac{1}{B} \mathbf{x}\right)-\pi^{n} K^{2} \ll_{C} B^{2 n-C} \pi^{-n+C} p^{-2} q^{-2},
$$

so letting

$$
\varepsilon_{1}=K \varepsilon_{0}+B^{2 n-C} \pi^{-n+C} p^{-2} q^{-2},
$$

we have proved (ii).

REMARK 4.1. $\Delta(\mathbf{y})$ measures the deviation of the weighted number of solutions to the two simultaneous congruences $p q|f(\mathbf{x}), p q| f(\mathbf{x}+\pi \mathbf{y})$ from its expected value. Unlike in the papers by Heath-Brown [12] and the author [19, we keep both congruence conditions in $\Delta(\mathbf{y})$ instead of using just the differenced polynomial $f(\mathbf{x}+\pi \mathbf{y})-f(\mathbf{x})$. This is the approach introduced by Salberger [20].

By Remark 2.1 and the properties $\left(\mathrm{R}_{0}(p)\right)$ and $\left(\mathrm{R}_{0}(q)\right)$, neither of $F_{q}^{\mathbf{y}}$ and $F_{p}^{\mathbf{y}}$ is identically zero. This means that $f^{\pi \mathbf{y}}$ is a polynomial of degree 
$d-1$ with leading form $\pi F^{\mathbf{y}}$, and moreover

$$
\operatorname{dim} Z_{q, \mathbf{y}}=\operatorname{dim} Z_{p, \mathbf{y}}=n-3 .
$$

Let

$$
X_{\mathbf{y}}=\operatorname{Spec} \mathbb{Z}\left[x_{1}, \ldots, x_{n}\right] /\left(f, f^{\pi \mathbf{y}}\right) .
$$

Now we write

$$
\Delta(\mathbf{y})=S(\mathbf{y})+\varepsilon_{2}(\mathbf{y})
$$

where we have defined

$$
\begin{aligned}
& S(\mathbf{y})=\sum_{\substack{\mathbf{v} \in \mathbb{F}_{p}^{n} \\
f_{p}(\mathbf{v})=f_{p}^{\pi \mathbf{y}}(\mathbf{v})=0}}\left(\sum_{\substack{\mathbf{x} \equiv \mathbf{v}(p) \\
q|f(\mathbf{x}) \\
q| f^{\pi \mathbf{y}}(\mathbf{x})}} W_{\pi \mathbf{y}}\left(\frac{1}{B} \mathbf{x}\right)-K(\mathbf{y})\right), \\
& \mathcal{E}_{2}(\mathbf{y})=\sum_{\substack{\mathbf{v} \in \mathbb{F}_{p}^{n} \\
f_{p}(\mathbf{v})=f_{p}^{\pi \mathbf{y}}(\mathbf{v})=0}} K(\mathbf{y})-p^{-2} q^{-2} \sum_{\mathbf{x} \in \mathbb{Z}^{n}} W_{\pi \mathbf{y}}\left(\frac{1}{B} \mathbf{x}\right),
\end{aligned}
$$

with

$$
K(\mathbf{y}):=p^{-n} q^{-2} \sum_{\mathbf{x} \in \mathbb{Z}^{n}} W_{\pi \mathbf{y}}\left(\frac{1}{B} \mathbf{x}\right) .
$$

But then

$$
\mathcal{E}_{2}(\mathbf{y})=K(\mathbf{y})\left(\# X_{\mathbf{y}}\left(\mathbb{F}_{p}\right)-p^{n-2}\right),
$$

and by Proposition 3.2 we have $\# X_{\mathbf{y}}\left(\mathbb{F}_{p}\right)=p^{n-2}+O\left(p^{\left(n+s_{p}(\mathbf{y})\right) / 2}\right)$, yielding

$$
\mathcal{E}_{2}(\mathbf{y}) \ll B^{n} p^{-\left(n-s_{p}(\mathbf{y})\right) / 2} q^{-2} .
$$

Thus we turn now to $S(\mathbf{y})$. We again apply Cauchy's inequality, using Proposition 3.1 to estimate the number of $\mathbb{F}_{p}$-points on $X_{\mathbf{y}}$. Thus we get

$$
S(\mathbf{y})^{2} \ll p^{n-2} \Sigma(\mathbf{y})
$$

where

$$
\Sigma(\mathbf{y})=\sum_{\mathbf{v} \in \mathbb{F}_{p}^{n}}\left(\sum_{\substack{\mathbf{x} \equiv \mathbf{v}(p) \\ q|f(\mathbf{x}) \\ q| f^{\pi \mathbf{y}}(\mathbf{x})}} W_{\pi \mathbf{y}}\left(\frac{1}{B} \mathbf{x}\right)-K(\mathbf{y})\right)^{2} .
$$

REMARK 4.2. In this second differencing step, our approach is intermediate between that of Heath-Brown and that of Salberger. Indeed, we shall complete the sum $(\bmod q)$, as in Heath-Brown's original argument [12, with respect to one of the two polynomials involved. This leads us to consider the closed subscheme defined by the three polynomials $f, f^{p \mathbf{z}}, f^{\pi \mathbf{y}, p \mathbf{z}}$ rather than the one defined by the four polynomials $f, f^{\pi \mathbf{y}}, f^{p \mathbf{z}}, f^{\pi \mathbf{y}}, p \mathbf{z}$. The reason is that the geometric results of [20] extend more readily in the former case. 
We have

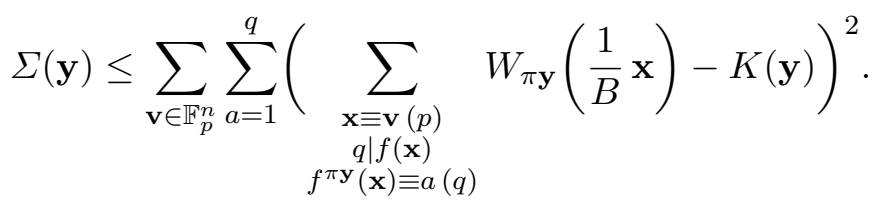

Denote by $\Sigma^{\prime}(\mathbf{y})$ the right hand side of 17 . Expanding the square, we get

$$
\left.\Sigma^{\prime}(\mathbf{y})=\sum_{\mathbf{v} \in \mathbb{F}_{p}^{n}} \sum_{\substack { a=1 \\
\begin{subarray}{c}{\mathbf{x} \equiv \mathbf{v}(p) \\
q \mid f(\mathbf{x}) \\
f^{\pi \mathbf{y}}(\mathbf{x}) \equiv a(q){ a = 1 \\
\begin{subarray} { c } { \mathbf { x } \equiv \mathbf { v } ( p ) \\
q | f ( \mathbf { x } ) \\
f ^ { \pi \mathbf { y } } ( \mathbf { x } ) \equiv a ( q ) } }\end{subarray}} W_{\pi \mathbf{y}}\left(\frac{1}{B} \mathbf{x}\right)\right)^{2}-2 K(\mathbf{y}) N_{W_{\pi \mathbf{y}}}(f, B, q)+p^{n} q K(\mathbf{y})^{2} .
$$

By Remark 3.1 we have the estimate

$$
N_{W_{\pi \mathbf{y}}}(f, B, q)=p^{n} q K(\mathbf{y})+O\left(B q^{(n-2) / 2}\right),
$$

insertion of which yields

$$
\begin{aligned}
\Sigma^{\prime}(\mathbf{y})= & \sum_{\mathbf{v} \in \mathbb{F}_{p}^{n}} \sum_{a=1}^{q}\left(\sum_{\substack{\mathbf{x} \equiv \mathbf{v}(p) \\
q \mid f(\mathbf{x}) \\
f^{\pi \mathbf{y}}(\mathbf{x}) \equiv a(q)}} W_{\pi \mathbf{y}}\left(\frac{1}{B} \mathbf{x}\right)\right)^{2}-p^{n} q K(\mathbf{y})^{2} \\
& +O\left(B^{n+1} p^{-n} q^{(n-6) / 2}\right) .
\end{aligned}
$$

As before, we proceed to expand the sum of squares as a double sum, introducing a third variable $\mathbf{z}$ :

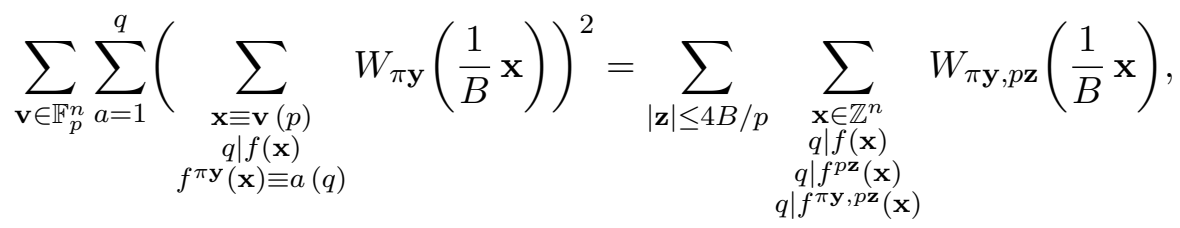

and then we compare the sum over $\mathbf{x}$ to its expected value $\Delta(\mathbf{y}, \mathbf{z})$. Another application of Lemma 3.1 yields

$$
q^{-3} \sum_{|\mathbf{z}| \leq 4 B / p} \sum_{\mathbf{x} \in \mathbb{Z}^{n}} W_{\pi \mathbf{y}, p \mathbf{z}}\left(\frac{1}{B} \mathbf{x}\right)-p^{n} q K(\mathbf{y})^{2} \ll_{C} B^{2 n-C} p^{-n+C} q^{-3},
$$

so it follows, in view of $15-18$, that

$$
\Delta(\mathbf{y}) \ll p^{(n-2) / 2}\left(\sum_{|\mathbf{z}| \leq 4 B / p} \Delta(\mathbf{y}, \mathbf{z})\right)^{1 / 2}+\mathcal{E}_{2}(\mathbf{y})+\mathcal{E}_{3},
$$

where

$$
\mathcal{E}_{3} \ll_{C} B^{(n+1) / 2} p^{-1} q^{(n-6) / 4}+B^{n-C} p^{-1+C} q^{-3 / 2} \quad \text { for any } C>0 .
$$

This proves (iii). 
Finally, we have

$$
\Delta(\mathbf{0})=N_{W_{\pi \mathbf{y}}}(f, B, p q)-p^{-2} q^{-2} \sum_{\mathbf{x} \in \mathbb{Z}^{n}} W_{\pi \mathbf{y}}\left(\frac{1}{B} \mathbf{x}\right),
$$

so arguing as in (14), we get (iv).

By $10-12$ we are led to evaluate the quantity

$$
\mathcal{E}_{4}:=\pi^{(n-1) / 2} p^{(n-2) / 4}\left(\sum_{\mathbf{y} \in \mathbb{Z}^{n} \backslash\{\mathbf{0}\}}\left(\sum_{\mathbf{z} \in \mathbb{Z}^{n}}|\Delta(\mathbf{y}, \mathbf{z})|\right)^{1 / 2}\right)^{1 / 2},
$$

which will be the strongest competitor to the main term in 107 . We shall derive an estimate for $\mathcal{E}_{4}$, subject to additional hypotheses. We maintain the convention that implied constants depend only on $n$ and $d$, unless otherwise specified.

Lemma 4.2. In addition to the hypotheses preceding Lemma 4.1, suppose that $B \geq q^{1 / 2}$ and $q \nmid(d-1)$. Then we have the estimate

$$
\begin{aligned}
\mathcal{E}_{4} \ll & B^{(3 n+1) / 4} \pi^{-1 / 2} p^{-1 / 2} q^{(n-4) / 8}+B^{(3 n+1) / 4} \pi^{(n-5) / 4} p^{-1 / 2} q^{-1 / 8} \\
& +B^{(3 n+1) / 4} \pi^{-1 / 2} p^{(n-5) / 4} q^{-1 / 8}+B^{3 n / 4} \pi^{(n-3) / 4} p^{-1 / 4} q^{-1 / 2} \\
& +B^{(n+1) / 2} \pi^{(n-3) / 4} p^{-1 / 4} q^{(n-4) / 8}+B^{3 n / 4} \pi^{(n-4) / 4} q^{-1 / 2} \\
& +B^{(2 n+3) / 4} \pi^{(n-4) / 4} q^{(n-5) / 8}+B^{(2 n+3) / 4} \pi^{(n-4) / 4} p^{(n-4) / 4} q^{-1 / 8} \\
& +B^{3 n / 4} \pi^{-1 / 2} p^{(n-2) / 4} q^{-1 / 4}+B^{(2 n+1) / 4} \pi^{-1 / 2} p^{(n-2) / 4} q^{(n-2) / 8} .
\end{aligned}
$$

REMARK. If we removed the hypothesis $B \geq q^{1 / 2}$, then we would get even more terms in (21). The hypothesis $q \nmid(d-1)$ ensures that Lemma 2.7(ii) is applicable.

Proof. We wish to switch the order of summation in 20). Thus we apply Hölder's inequality [7, Theorem 11] to get

$$
\sum_{\mathbf{y} \in \mathbb{Z}^{n} \backslash\{\mathbf{0}\}}\left(\sum_{\mathbf{z} \in \mathbb{Z}^{n}}|\Delta(\mathbf{y}, \mathbf{z})|\right)^{1 / 2} \ll\left(\frac{B}{\pi}\right)^{n / 2}\left(\sum_{\mathbf{y} \in \mathbb{Z}^{n} \backslash\{\mathbf{0}\}} \sum_{\mathbf{z} \in \mathbb{Z}^{n}}|\Delta(\mathbf{y}, \mathbf{z})|\right)^{1 / 2} .
$$

Here we have used the fact that $\Delta(\mathbf{y}, \mathbf{z})$ vanishes identically for $|\mathbf{y}| \geq 4 B / \pi$. Then (20) yields

$$
\mathcal{E}_{4} \ll B^{n / 4} \pi^{(n-2) / 4} p^{(n-2) / 4}\left(\sum_{(\mathbf{y}, \mathbf{z}) \in \mathcal{B}}|\Delta(\mathbf{y}, \mathbf{z})|\right)^{1 / 4},
$$

where the domain of summation is defined by

$$
\mathcal{B}=\left\{(\mathbf{y}, \mathbf{z}) \in \mathbb{Z}^{n} \times \mathbb{Z}^{n} ;|\mathbf{y}| \leq \frac{4 B}{\pi},|\mathbf{z}| \leq \frac{4 B}{p}, \mathbf{y} \neq \mathbf{0}\right\} .
$$


To calculate $\mathcal{E}_{4}$, we shall partition $\mathcal{B}$ into three subsets

$$
\begin{aligned}
& \mathcal{B}_{1}=\left\{(\mathbf{y}, \mathbf{z}) \in \mathcal{B} ; \operatorname{dim} Z_{q, \mathbf{y}, \mathbf{z}}=n-4\right\}, \\
& \mathcal{B}_{2}=\left\{(\mathbf{y}, \mathbf{z}) \in \mathcal{B} ; \operatorname{dim} Z_{q, \mathbf{y}, \mathbf{z}}>n-4, \mathbf{z} \neq \mathbf{0}\right\}, \\
& \mathcal{B}_{3}=\{(\mathbf{y}, \mathbf{z}) \in \mathcal{B} ; \mathbf{z}=\mathbf{0}\},
\end{aligned}
$$

and let

$$
\mathcal{E}_{4, i}=B^{n / 4} \pi^{(n-2) / 4} p^{(n-2) / 4}\left(\sum_{(\mathbf{y}, \mathbf{z}) \in \mathcal{B}_{i}}|\Delta(\mathbf{y}, \mathbf{z})|\right)^{1 / 4}
$$

for $i \in\{1,2,3\}$.

We start with $\mathcal{E}_{4,1}$. Let us partition $\mathcal{B}_{1}$ even further into subsets (some of them possibly empty)

$$
\mathcal{B}_{1, \sigma, s}=\left\{(\mathbf{y}, \mathbf{z}) \in \mathcal{B}_{1} ; \sigma_{q}(\mathbf{z})=\sigma, s_{q}(\mathbf{y}, \mathbf{z})=s\right\},
$$

where $s \in\{-1, \ldots, n-4\}, \sigma \in\{-1, \ldots, n-1\}$ and $\sigma_{q}(\cdot)$ and $s_{q}(\cdot, \cdot)$ are as defined in Notation 4.1. Using [19, Thm. 3.3] (combined with Remark 3.1 as usual) we have the estimate

$$
|\Delta(\mathbf{y}, \mathbf{z})| \ll B^{s+2} q^{(n-5-s) / 2}
$$

for $(\mathbf{y}, \mathbf{z}) \in \mathcal{B}_{1, \sigma, s}$. Recall that $F$ satisfies $\left(\mathrm{R}_{1}(q)\right)$ and $\left(\mathrm{R}_{2}(q)\right)$. This implies that

$$
\operatorname{dim} T_{\sigma}\left(Z_{q}\right) \leq n-2-\sigma
$$

for $\sigma \in\{-1, \ldots, n-1\}$. Furthermore, if $\mathbf{z} \in \mathbb{Z}^{n}$ satisfies $\sigma_{q}(\mathbf{z})=\sigma$, then

$$
\operatorname{dim} T_{s, \mathbf{z}}\left(Z_{q}\right) \leq n-1-s+\sigma
$$

for $s \geq \sigma$. Applying Proposition 3.1. we get

$$
\# \mathcal{B}_{1, \sigma, s} \ll \begin{cases}\left(\frac{B}{p}\right)^{n-1-\sigma}\left(\frac{B}{\pi}\right)^{n-s+\sigma} & \text { if } s \geq \sigma, \\ \left(\frac{B}{p}\right)^{n-1-\sigma}\left(\frac{B}{\pi}\right)^{n} & \text { if } s<\sigma .\end{cases}
$$

Combining (23) and (24), we have

$$
\sum_{(\mathbf{y}, \mathbf{z}) \in \mathcal{B}_{1, \sigma, s}}|\Delta(\mathbf{y}, \mathbf{z})| \ll U_{\sigma, s}
$$

where

$$
U_{\sigma, s}:= \begin{cases}B^{2 n+1} \pi^{-n+s-\sigma} p^{-n+1+\sigma} q^{(n-5-s) / 2} & \text { if } s \geq \sigma, \\ B^{2 n+s-\sigma+1} \pi^{-n} p^{-n+1+\sigma} q^{(n-5-s) / 2} & \text { if } s<\sigma .\end{cases}
$$

By carefully examining the relations between the quantities $U_{\sigma, s}$, one sees that 


$$
\begin{aligned}
\sum_{(\mathbf{y}, \mathbf{z}) \in \mathcal{B}_{1}}|\Delta(\mathbf{y}, \mathbf{z})| \ll & \sum_{\sigma, s} U_{\sigma, s} \ll U_{-1,-1}+U_{-1, n-4}+U_{n-4, n-4} \\
= & B^{2 n+1} \pi^{-n} p^{-n} q^{(n-4) / 2}+B^{2 n+1} \pi^{-3} p^{-n} q^{-1 / 2} \\
& +B^{2 n+1} \pi^{-n} p^{-3} q^{-1 / 2} .
\end{aligned}
$$

We conclude that

$$
\begin{aligned}
\mathcal{E}_{4,1} \ll & B^{(3 n+1) / 4} \pi^{-1 / 2} p^{-1 / 2} q^{(n-4) / 8}+B^{(3 n+1) / 4} \pi^{(n-5) / 4} p^{-1 / 2} q^{-1 / 8} \\
& +B^{(3 n+1) / 4} \pi^{-1 / 2} p^{(n-5) / 4} q^{-1 / 8} .
\end{aligned}
$$

Next, we turn our attention to $\mathcal{E}_{4,2}$. For $(\mathbf{y}, \mathbf{z}) \in \mathcal{B}_{2}$, we cannot apply the case $r=3$ of Remark 3.1 to estimate $|\Delta(\mathbf{y}, \mathbf{z})|$ as in $(23)$, since $\operatorname{dim} Z_{q, \mathbf{y}, \mathbf{z}}>$ $n-4$. However, since we assume that $F$ has property $\left(\mathrm{R}_{0}(q)\right)$, we do know, by Lemma 2.6 , that $\operatorname{dim} Z_{q, \mathbf{z}}=n-3$. Thus, it follows from the case $r=2$ of Remark 3.1 that

$$
\begin{aligned}
|\Delta(\mathbf{y}, \mathbf{z})| \leq & N_{W_{\pi \mathbf{y}, p \mathbf{z}}}\left(f, f^{p \mathbf{z}}, B, q\right)+O\left(B^{n} q^{-3}\right) \\
= & q^{-2} N_{W_{\pi \mathbf{y}, p \mathbf{z}}}(0, B, q)+O\left(B^{\sigma_{q}(\mathbf{z})+2} q^{\left(n-4-\sigma_{q}(\mathbf{z})\right) / 2}\right) \\
& +O\left(B^{n} q^{-3}\right) \\
\ll & B^{n} q^{-2}+B^{\sigma_{q}(\mathbf{z})+2} q^{\left(n-4-\sigma_{q}(\mathbf{z})\right) / 2} .
\end{aligned}
$$

Here we have used the fact that $\sigma_{q}(\mathbf{z}) \geq s_{q}(\mathbf{z})$, so that $\left(B q^{-1 / 2}\right)^{\sigma_{q}(\mathbf{z})} \geq$ $\left(B q^{-1 / 2}\right)^{s_{q}(\mathbf{z})}$.

We shall partition $\mathcal{B}_{2}$ into subsets

$$
\mathcal{B}_{2, \sigma}=\left\{(\mathbf{y}, \mathbf{z}) \in \mathcal{B}_{2} ; \sigma_{q}(\mathbf{z})=\sigma\right\}
$$

where $\sigma \in\{-1, \ldots, n-1\}$. Note that $\mathcal{B}_{2, n-2}=\mathcal{B}_{2, n-1}=\emptyset$ since $\operatorname{dim} Z_{q, \mathbf{z}}=$ $n-3$, and $\mathcal{B}_{2,-1}=\emptyset$ by Lemma 2.7(ii) (recall that $\mathbf{y} \neq \mathbf{0} \neq \mathbf{z}$ if $(\mathbf{y}, \mathbf{z}) \in \mathcal{B}_{2}$ ). Furthermore, Lemma 2.7(ii), property $\left(\mathrm{R}_{1}(q)\right)$ and Proposition 3.1 imply that

$$
\# \mathcal{B}_{2,0} \ll\left(\frac{B}{p}\right)^{n-1}\left(\frac{B}{\pi}\right) \text { and } \quad \# \mathcal{B}_{2, \sigma} \ll\left(\frac{B}{p}\right)^{n-1-\sigma}\left(\frac{B}{\pi}\right)^{2}
$$

for $\sigma \in\{1, \ldots, n-3\}$. Inserting (26), we get

$$
\sum_{(\mathbf{y}, \mathbf{z}) \in \mathcal{B}_{2, \sigma}}|\Delta(\mathbf{y}, \mathbf{z})| \ll V_{\sigma, 1}+V_{\sigma, 2}
$$

for $\sigma \in\{0, \ldots, n-3\}$, where

$$
V_{\sigma, 1}= \begin{cases}B^{2 n} \pi^{-1} p^{-n+1} q^{-2} & \text { if } \sigma=0, \\ B^{2 n+1-\sigma} \pi^{-2} p^{-n+1+\sigma} q^{-2} & \text { if } 1 \leq \sigma \leq n-3,\end{cases}
$$




$$
V_{\sigma, 2}= \begin{cases}B^{n+2} \pi^{-1} p^{-n+1} q^{(n-4) / 2} & \text { if } \sigma=0, \\ B^{n+3} \pi^{-2} p^{-n+1+\sigma} q^{(n-4-\sigma) / 2} & \text { if } 1 \leq \sigma \leq n-3 .\end{cases}
$$

As in the calculation of $\mathcal{E}_{4,1}$, some of the $V_{\sigma, i}$ can be neglected when estimating $\mathcal{E}_{4,2}$. We have

$$
\sum_{(\mathbf{y}, \mathbf{z}) \in \mathcal{B}_{2}}|\Delta(\mathbf{y}, \mathbf{z})| \ll V_{0,1}+V_{0,2}+V_{1,1}+V_{1,2}+V_{n-3,2},
$$

which implies that

$$
\begin{aligned}
\mathcal{E}_{4,2} \ll & B^{3 n / 4} \pi^{(n-3) / 4} p^{-1 / 4} q^{-1 / 2}+B^{(n+1) / 2} \pi^{(n-3) / 4} p^{-1 / 4} q^{(n-4) / 8} \\
& +B^{3 n / 4} \pi^{(n-4) / 4} q^{-1 / 2}+B^{(2 n+3) / 4} \pi^{(n-4) / 4} q^{(n-5) / 8} \\
& +B^{(2 n+3) / 4} \pi^{(n-4) / 4} p^{(n-4) / 4} q^{-1 / 8} .
\end{aligned}
$$

Finally, we calculate $\mathcal{E}_{4,3}$. For $(\mathbf{y}, \mathbf{0}) \in \mathcal{B}_{3}$, we estimate $|\Delta(\mathbf{y}, \mathbf{0})|$ using the case $r=1$ of Remark 3.1 .

$$
|\Delta(\mathbf{y}, \mathbf{z})| \leq N_{W_{\pi \mathbf{y}, p \mathbf{z}}}(f, B, q)+O\left(B^{n} q^{-3}\right) \ll B^{n} q^{-1}+B q^{(n-2) / 2} .
$$

Since $\# \mathcal{B}_{3} \ll(B / \pi)^{n}$, we get

$$
\mathcal{E}_{4,3} \ll B^{3 n / 4} \pi^{-1 / 2} p^{(n-2) / 4} q^{-1 / 4}+B^{(2 n+1) / 4} \pi^{-1 / 2} p^{(n-2) / 4} q^{(n-2) / 8} .
$$

Putting together the contributions from (25), (27) and (28), we arrive at the estimate (21).

5. Proof of the main theorems. Let $f$ be a polynomial in $\mathbb{Z}\left[x_{1}, \ldots, x_{n}\right]$ of degree $d \geq 4$ with leading form $F$, let $Z=\operatorname{Proj} \mathbb{Z}\left[x_{1}, \ldots, x_{n}\right] /(F)$, and suppose that $Z_{\mathbb{Q}}$ is a non-singular subscheme of $\mathbb{P}_{\mathbb{Q}}^{n-1}$. Note that Lemma 4.1(i) gives an asymptotic formula for $N_{W}(f, B, \pi p q)$. However, we shall only use it as an upper bound, and try to deduce a good upper bound for $N(f, B)$ by choosing $\pi, p$ and $q$ wisely in terms of $B$. It turns out that the following relations are desirable:

$$
\begin{aligned}
& \pi \asymp B^{\left(n^{2}-n-2\right) /\left(n^{2}+8 n-4\right)}, \\
& p \asymp B^{\left(n^{2}-2 n+8\right) /\left(n^{2}+8 n-4\right)}, \\
& q \asymp B^{2\left(n^{2}-n-2\right) /\left(n^{2}+8 n-4\right)},
\end{aligned}
$$

since then the first, second and ninth terms in 21) will be of the same order of magnitude as the main term in (10), and all other terms involved will be smaller. To be able to use Lemma 4.1 we need to have $q \gg B$, which is consistent with (29) as soon as $n \geq 10$. However, in case $n<10$, the estimate in Theorem 1.1 already follows from [12, Thm. 2]. More importantly, the results of Lemma 4.1 are subject to a set of hypotheses (9) on $\pi, p, q$. We need to show that such $\pi, p, q$ exist in the specified intervals 29 . 
By Corollary 2.1, however, the set of primes not fulfilling these criteria is finite. Thus, Bertrand's postulate [8, Theorem 418] ensures that the intervals specified in (29), with implied constants depending on $F$, contain primes satisfying (9). We are thus allowed to insert (29) into Lemmata 4.1 and 4.2 . Then we have, for the main term in (10),

$$
(\pi p q)^{-1} N_{W}(0, B, \pi p q) \ll B^{n}(\pi p q)^{-1} \ll_{F} B^{n-4+(37 n-18) /\left(n^{2}+8 n-4\right)} .
$$

The same holds for the "main" auxiliary term — by Lemma 4.2 we have

$$
\mathcal{E}_{4} \ll{ }_{F} B^{n-4+(37 n-18) /\left(n^{2}+8 n-4\right)},
$$

where, as mentioned above, the first, second and ninth terms in 21 dominate the expression. Thus, to finish the proof of Theorem 1.1 it remains to check that the remaining error terms occurring in Lemma 4.1 are small enough. We shall omit these calculations.

The key argument in the proof of Theorem 1.2 is the following lemma, which is a version of a result by Heath-Brown (see [13, Thm. 4] and [2, Lemma 5]).

Lemma 5.1. Let $f \in \mathbb{Z}\left[x_{1}, \ldots, x_{n}\right]$ be a polynomial of degree $d$. Let

$$
S(f, B)=\left\{\mathbf{x} \in \mathbb{Z}^{n} ; f(\mathbf{x})=0,|\mathbf{x}| \leq B\right\} .
$$

Then one of the following holds:

(i) There is a constant $\theta$, depending only on $n$ and $d$, with $\|f\| \ll_{n, d} B^{\theta}$.

(ii) There exists a polynomial $g \in \mathbb{Z}\left[x_{1}, \ldots, x_{n}\right]$ of degree $d$, not equal to $\lambda f$ for any $\lambda \in \mathbb{Q}$, such that $g(\mathbf{x})=0$ for every $\mathbf{x} \in S(f, B)$.

Proof. The result follows upon applying [2, Lemma 5] to the homogenization $F_{0} \in \mathbb{Z}\left[x_{0}, \ldots, x_{n}\right]$ of $f$.

Now we are able to prove Theorem 1.2. Suppose that (i) holds in Lemma 5.1. Then by Corollary 2.1 we have

$$
\prod_{p \in \mathcal{P}(F)} p \ll_{n, d} B^{\theta \kappa} .
$$

Thus it is possible, using Bertrand's postulate, to find primes $\pi, p$ and $q$ satisfying (9) and (29), with the implied constants in (29) depending only on $n$ and $d$. Now we proceed exactly as in the proof of Theorem 1.1, except that all implied constants now depend only on $n$ and $d$. We get the following result.

TheOREM 5.1 (Theorem 1.1). Under the hypotheses of Theorem 1.1, suppose furthermore that there is a constant $\theta \ll_{n, d} 1$ such that $\|f\| \ll_{n, d} B^{\theta}$. Then

$$
N(f, B) \ll_{n, d} B^{n-4+(37 n-18) /\left(n^{2}+8 n-4\right)} .
$$


In case (ii) one easily sees that $N(f, B) \ll_{n, d} B^{n-2}$. To improve this to $B^{n-3+\varepsilon}$ requires some work, to which we devote Section 6 . The estimate given by Theorem 6.1 is enough to deduce Theorem 1.2 .

6. Integral points on certain affine varieties. The aim of this section is to prove the following result.

TheOrem 6.1. Let $n \geq 4$. Let $f \in \mathbb{Z}\left[x_{1}, \ldots, x_{n}\right]$ be a polynomial of degree $d \geq 4$, whose leading form $F$ defines a non-singular hypersurface in $\mathbb{P}_{\mathbb{Q}}^{n-1}$. Let $g \in \mathbb{Z}\left[x_{1}, \ldots, x_{n}\right]$ be another polynomial, not divisible by $f$. Then

$$
N(f, g, B) \ll_{n, d, \varepsilon} \begin{cases}B^{n-3+1 / 12+\varepsilon}, & 4 \leq n \leq 11 \\ B^{n-3+\varepsilon}, & n \geq 12 .\end{cases}
$$

First we make some remarks on notation. Unless otherwise stated, we work over $\mathbb{Q}$, that is, $\mathbb{A}^{n}=\mathbb{A}_{\mathbb{Q}}^{n}$ and $\mathbb{P}^{n}=\mathbb{P}_{\mathbb{Q}}^{n}$. We shall use the notation $V\left(\gamma_{1}, \ldots, \gamma_{r}\right)$ for the closed subset of $\mathbb{A}^{n}$ defined by $\gamma_{1}=\cdots=\gamma_{r}=0$, endowed with its reduced scheme structure. We denote by $H_{0} \subset \mathbb{P}^{n}$ the hyperplane defined by $x_{0}=0$. If $U \subseteq \mathbb{A}^{n}$ is a locally closed subset, then we define

$$
U(\mathbb{Z}, B)=U(\mathbb{Q}) \cap \mathbb{Z}^{n} \cap[-B, B]^{n}
$$

for any positive real number $B$, and $N(U, B)=\# U(\mathbb{Z}, B)$.

In proving Theorem 6.1 we shall use results by Browning, Heath-Brown and Salberger [2]. However, we shall need a slightly more general version of [2, Thm. 2], which was shown to us by Salberger.

TheOREM 6.2. Let $f \in \overline{\mathbb{Q}}\left[x_{1}, x_{2}, x_{3}\right]$ be an irreducible polynomial of degree $d \geq 3$. Suppose that the leading form $F$ of $f$ has no irreducible factors of degree 1 or 2 . Then

$$
N(f, B) \ll_{d, \varepsilon} \begin{cases}B^{5 /(3 \sqrt{3})+1 / 4+\varepsilon}, & d=3, \\ B^{3 /(2 \sqrt{d})+1 / 3+\varepsilon}, & d=4 \text { or } 5, \\ B^{1+\varepsilon}, & d \geq 6 .\end{cases}
$$

We achieve this generalization by noting that the hypotheses in 2, Lemma 9] can be weakened. In the statement of that lemma, it suffices to assume that $X \cap H$ has no irreducible component of degree at most $e$. Indeed, that is enough to provoke the contradiction in the last line of the proof of the lemma.

From this we immediately get the following strengthening of [2, Prop. 1].

Proposition 6.1. Let $X \subset \mathbb{P}_{\overline{\mathbb{Q}}}^{3}$ be an integral surface of degree $d \geq 3$ such that every irreducible component of $X \cap H_{0}$ (with its reduced scheme structure) has degree at least 3 . Let $D=1$ or 2 , let $I_{D}$ be any finite set of 
integral curves $C \subset X$ of degree $D$, and let $\Sigma=\bigcup_{I_{D}} C$. Then

$$
\begin{aligned}
& \#\left\{\left(x_{1}, x_{2}, x_{3}\right) \in \mathbb{Z}^{3} \cap[-B, B]^{3} ;\left(1: x_{1}, x_{2}, x_{3}\right) \in \Sigma(\mathbb{Q})\right\} \\
& \ll_{d, \varepsilon} B^{\varepsilon} \max \left\{B^{2 / d}, B^{1 / D}, \# I_{D}\right\} .
\end{aligned}
$$

In the proof of [2, Thm. 2], one uses Heath-Brown's determinant method to prove that the integral points of height at most $B$ on the surface $S$ defined by $f=0$ are contained in $O_{d, \varepsilon}\left(B^{2 / \sqrt{d}}\right)$ curves on $S$ of bounded degree. It is only in handling the contribution from lines and conics that the irreducibility of $F$ is used. For the rest of the proof, it suffices to assume that $f$ itself is irreducible. Thus, letting Proposition 6.1 play the role of [2, Prop. 1] in the proof of [2, Thm. 2], we immediately deduce Theorem 6.2.

Proof of Theorem 6.1. Let $X=V(f) \subset \mathbb{A}^{n}$ and let $Y$ be any integral component of $V(f, g) \subset \mathbb{A}^{n}$. Note that $X$ is geometrically integral by assumption, so the dimension of $Y$ is $n-2$. We shall prove that

$$
N(Y, B) \ll_{n, d, \varepsilon} \begin{cases}B^{n-3+1 / 12+\varepsilon}, & 4 \leq n \leq 11, \\ B^{n-3+\varepsilon}, & n \geq 12 .\end{cases}
$$

Let $\bar{X}, \bar{Y} \subset \mathbb{P}^{n}$ be the respective projective closures. Let $X_{0}=\bar{X} \cap H_{0}$ and $Y_{0}=\bar{Y} \cap H_{0}$. Our hypothesis implies that $X_{0}$ is non-singular. Then, as observed in [21, Lemma 6.2], any closed subscheme of $\left(X_{0}\right)_{\overline{\mathbb{Q}}}$ of pure codimension one is the intersection of $\left(X_{0}\right)_{\overline{\mathbb{Q}}}$ with a hypersurface $G \subset \mathbb{P}^{n-1}$. This is a consequence of the Noether-Lefschetz theorem (use [10, Cor. 3.3, p. 180] and the following exercises). Thus, every integral component of $\left(Y_{0}\right)_{\overline{\mathbb{Q}}}$ has degree divisible by $d$, and in particular $d \mid \operatorname{deg} Y$.

Let $d^{\prime}=\operatorname{deg} Y$. We can assume that $Y$ is geometrically integral. Indeed, in case $Y$ is integral, but not geometrically integral, one can argue as in the proof of [21, Thm. 2.1] to conclude that all rational points on $Y$ lie on a proper subvariety, obtained as the intersection of all the irreducible components of $Y_{\overline{\mathbb{Q}}}$. Thus we can use a trivial estimate (e.g. Proposition 3.1 for suitably chosen $q$ ) to conclude that

$$
N(Y, B) \ll_{n, d} B^{n-3} .
$$

We have the following result, which we shall prove in Section 6.1.

Proposition 6.2. Let $X \subset \mathbb{A}_{\overline{\mathbb{Q}}}^{n}$ be an integral closed subvariety of dimension $m \leq n-2$ and degree $d$. Let $\bar{X} \subset \mathbb{P}_{\overline{\mathbb{Q}}}^{n}$ be its projective closure and $X_{0}=\bar{X} \cap H_{0}$. Then there exists an $((m+1) \times n)$-matrix $A$ and an $(m+1)$-vector $\mathbf{b}$, with integer entries of size $O_{n, d}(1)$, such that the morphism $\pi: X \rightarrow \mathbb{A}_{\overline{\mathbb{Q}}}^{m+1}$ given by $\mathbf{x} \mapsto A \mathbf{x}+\mathbf{b}$ is birational onto its image. In particular, its fibres consist of at most d points, and $\pi(X)$ is an integral closed hypersurface of degree $d$. 
Moreover, it induces a morphism $\bar{\pi}: \bar{X} \rightarrow \mathbb{P}_{\overline{\mathbb{Q}}}^{m+1}$ with the following property. If

$$
X_{0}=X_{0,1} \cup \cdots \cup X_{0, k}
$$

is the decomposition of $X_{0}$ into irreducible components and $\operatorname{deg} X_{0, i}=d_{i}$, then $\bar{\pi}: X_{0, i} \rightarrow \mathbb{P}_{\overline{\mathbb{Q}}}^{m+1}$ is birational onto its image for each $i$. In particular,

$$
\bar{\pi}(X) \cap H_{0}=\bar{\pi}\left(X_{0}\right)=\bar{\pi}\left(X_{0,1}\right) \cup \cdots \cup \bar{\pi}\left(X_{0, k}\right),
$$

where each $\bar{\pi}\left(X_{0, i}\right)$ is integral of dimension $m-1$ and degree $d_{i}$.

Thus we find a geometrically integral hypersurface $W=\pi(Y) \subset \mathbb{A}^{n-1}$ of degree $d^{\prime}$ such that $N(Y, B) \leq d \cdot N(W, c B)$ for some constant $c \ll_{n, d} 1$, and such that if $\bar{W} \subset \mathbb{P}^{n-1}$ is the projective closure and $W_{0}=\bar{W} \cap H_{0}$ (taken with its reduced scheme structure), then $\left(W_{0}\right)_{\overline{\mathbb{Q}}}$ has no irreducible component of degree less than $d$.

It is a standard fact [2, Lemma 7] that we can find a hyperplane $H \subset$ $\mathbb{P}^{n-1}$, defined by a linear form with integer coefficients of size $O_{n, d}(1)$, such that the intersection of $H$ with $W$ or any of the irreducible components of $\left(W_{0}\right)_{\overline{\mathbb{Q}}}$ is again irreducible. Indeed, the set $\mathcal{E} \subset \check{\mathbb{P}}^{n-1}$ of hyperplanes $H$ such that this fails is a proper closed subscheme of degree $O_{n, d}(1)$. After a suitable change of variables (sending $H_{0}$ to itself), we can assume that $H$ is given by $x_{n-1}=0$. Letting $H_{a} \subset \mathbb{A}^{n-1}$ for any $a \in \mathbb{Z}$ be the hyperplane given by $x_{n-1}=a$, and putting $W_{a}=W \cap H_{a}$, we have

$$
N(W, B)=\sum_{a=-c^{\prime} B}^{c^{\prime} B} N\left(W_{a}, B\right) .
$$

For all but $O_{n, d}(1)$ values of $a, \bar{W}_{a}$ is geometrically irreducible, and $\bar{W}_{a} \cap H_{0}$ has no irreducible components over $\overline{\mathbb{Q}}$ of degree less than $d$. Indeed, let $\mathcal{H}$ be the linear pencil of hyperplanes $\lambda H+\mu H_{0}$ parameterized by $(\lambda: \mu) \in \mathbb{P}^{1}$. Since $\mathcal{H} \nsubseteq \mathcal{E}$, we have $\operatorname{dim}(\mathcal{H} \cap \mathcal{E})=0$. The exceptional values of $a$ yield an acceptable contribution to (30) by a trivial estimate for $N\left(W_{a}, B\right)$.

Applying this process inductively, much as in [2, §4], we find a collection of $O_{n, d}\left(B^{n-4}\right)$ geometrically irreducible surfaces $S \subset \mathbb{A}^{3}$ of degree $d^{\prime}$ such that the curve $S_{0}=\bar{S} \cap H_{0}$ has no components over $\overline{\mathbb{Q}}$ of degree less than $d$, and such that the estimate

$$
N(W, B) \leq \sum_{S} N\left(S, c^{\prime \prime} B\right)+O_{n, d}\left(B^{n-3}\right)
$$

holds for some constant $c^{\prime \prime} \ll_{n, d} 1$. There are now two cases to consider.

Case 1: $d^{\prime} \geq 2 d$. Then Theorem 6.2 yields the estimate

$$
N(S, B) \ll_{d, \varepsilon} B^{1+\varepsilon},
$$

which suffices to establish the desired bound for $N(Y, B)$. 
CASE $2: d^{\prime}=d$. Then the estimate given by Theorem 6.2 is

$$
N(Y, B) \ll_{n, d, \varepsilon} B^{n-3+1 / 12+\varepsilon} .
$$

For large $n$, we shall derive a better estimate by applying our main result inductively.

In the present case we necessarily have $Y_{0}=X_{0} \cap \Gamma_{0}$ for some hyperplane $\Gamma_{0} \subset \mathbb{P}^{n-1}$. But then we must also have $\bar{Y}=\bar{X} \cap \Gamma$ for some hyperplane $\Gamma \subset \mathbb{P}^{n}$. (Indeed, let $\mathcal{G}$ be the family of $\Gamma \in \mathbb{G}(n-1, n)$ such that $\Gamma_{0} \subset \Gamma$. Then $Y_{0} \subseteq \bar{Y} \cap \Gamma$ for every $\Gamma \in \mathcal{G}$, and the inclusion has to be strict for some $\Gamma$. If $\Gamma$ intersected $\bar{Y}$ properly, then we would have $\bar{Y} \cap \Gamma=Y_{0} \cup Z$ for some closed subscheme $Z \subset \bar{Y}$ of codimension one. But this would contradict the fact that $\operatorname{deg}(\bar{Y} \cap \Gamma)=d=\operatorname{deg} Y_{0}$. Thus we conclude that $\bar{Y} \subseteq \Gamma$.)

Thus, in this case we have $Y=X \cap L$ for some hyperplane $L \subset \mathbb{A}^{n}$. Now $\Lambda:=L \cap \mathbb{Z}^{n}$ is a lattice of dimension $r \leq n-1$. Thus, by [13, Lemma 1], it has a basis $\mathbf{b}_{1}, \ldots, \mathbf{b}_{r}$ such that for every $\mathbf{x}=\sum \lambda_{j} \mathbf{b}_{j} \in \Lambda \cap[-B, B]^{n}$ we have $\lambda_{j} \ll|\mathbf{x}| /\left|\mathbf{b}_{j}\right| \ll B$. Thus we get a bijection between $Y(\mathbb{Z}, B)$ and $Y^{\prime}(\mathbb{Z}, c B)$, where $c \ll_{n, d} 1, h(\mathbf{y})=f\left(\sum y_{j} \mathbf{b}_{j}\right)$ and $Y^{\prime}=V(h) \subset \mathbb{A}^{r}$.

By Lemma 5.1 we can assume that $\|h\| \ll_{n, d} B^{\theta}$, since in case (ii) we can use a trivial estimate as in (31) to conclude that

$$
N(Y, B) \leq N(h, c B) \ll_{n, d} B^{r-2} \leq B^{n-3} .
$$

Since $X_{0}$ is non-singular, it is well known [14, Appendix, Thm. 2] that $Y_{0}$ can have at most isolated singularities. Thus, the same holds for the closed subscheme $Y_{0}^{\prime} \subset \mathbb{P}^{r}$ defined by the leading form of $h$. Then we can find a hyperplane $\Pi \subset \mathbb{P}^{r}$, defined by a linear form with integer coefficients of size $O_{n, d}(1)$, such that $Y_{0}^{\prime} \cap \Pi$ is non-singular. For a proof of this "effective" version of Bertini's theorem, see [19, Lemma 2.8]. After a suitable linear transformation we may assume that $\Pi$ is given by $y_{r}=0$. For any $a \in \mathbb{Z}$, let $h_{a} \in \mathbb{Z}\left[y_{1}, \ldots, y_{r}\right]$ be given by $h_{a}\left(y_{1}, \ldots, y_{r-1}\right)=h\left(y_{1}, \ldots, y_{r-1}, a\right)$. All the polynomials $h_{a}$ then have the same non-singular leading form. Thus we can apply the estimate of Theorem 5.1 to get

$$
N\left(h_{a}, B\right) \ll_{n, d} B^{n-6+(37(n-2)-18) /\left((n-2)^{2}+8(n-2)-4\right)} .
$$

This yields an estimate

$$
N(Y, B) \leq N(h, c B) \ll_{n, d} \sum_{a=-c B}^{c B} N\left(h_{a}, c B\right) \ll_{n, d} B^{n-5+(37 n-92) /\left(n^{2}+4 n-16\right)} .
$$

As soon as $n \geq 12$, we get $N(Y, B) \ll_{n, d} B^{n-3}$.

6.1. Birational projections of bounded height. Let $Z \subset \mathbb{P}_{\overline{\mathbb{Q}}}^{n}$ be an integral closed subvariety of dimension $m \leq n-2$. Throughout this section, we work over $\overline{\mathbb{Q}}$, but henceforth we shall omit this subscript. Let $\Lambda$ be an 
$(n-m-2)$-plane and $\Gamma$ an $(m+1)$-plane such that $\Lambda \cap \Gamma=\emptyset$. We recall the construction of the projection

$$
\pi_{\Lambda, \Gamma}: \mathbb{P}^{n} \backslash \Lambda \rightarrow \Gamma
$$

from $\Lambda$ to $\Gamma$ (see [9, Lecture 3]). Identifying $\Gamma$ with $\mathbb{P}^{m+1}$, we write $\pi_{\Lambda}$ : $\mathbb{P}^{n} \rightarrow \mathbb{P}^{m+1}$. It is known that for a generic $\Lambda \in \mathbb{G}(n-m-2, n)$, the projection $\left.\pi_{\Lambda}\right|_{Z}: Z \rightarrow \mathbb{P}^{m+1}$ is birational onto its image. In particular, $\pi(Z)$ is integral and $\operatorname{deg} \pi_{\Lambda}(Z)=\operatorname{deg} Z$. In [2, §3] it is shown that one can also find such a projection where $\Lambda$ is defined over $\mathbb{Q}$ and of bounded height. We shall need an affine version of that statement.

Let us recall the notation of [2, §3]. Let $Z \subset \mathbb{P}^{n}$ be a closed subvariety of dimension $m$ and degree $d$. For any $\Lambda \in \mathbb{G}(n-m-2, n)$, define

$$
\begin{aligned}
S_{\Lambda, Z} & =\{M \in \mathbb{G}(n-m-1, n) ; \Lambda \subset M, \#(M \cap Z) \geq 2\}, \\
Y_{Z} & =\left\{\Lambda \in \mathbb{G}(n-m-2, n) ; \operatorname{dim} S_{\Lambda, Z} \geq m\right\}, \\
Y_{Z}^{\prime} & =\{\Lambda \in \mathbb{G}(n-m-2, n) ; \Lambda \cap Z \neq \emptyset\} .
\end{aligned}
$$

Then, in case $Z$ is integral, it is shown [2, Lemma 6] that $Y_{Z}$ and $Y_{Z}^{\prime}$ are proper closed subvarieties of $\mathbb{G}(n-m-2, n)$ of degree $O_{n, d}(1)$. Furthermore, as soon as $\Lambda \notin Y_{Z} \cup Y_{Z}^{\prime}$, the morphism $\pi_{\Lambda}: Z \rightarrow \mathbb{P}^{m+1}$ is birational onto its image, and its fibres consist of at most $d$ points.

Let now $X \subset \mathbb{A}^{n}$ be an integral closed subvariety of dimension $m$ and degree $d$, and let $Z \subset \mathbb{P}^{n}$ be its projective closure. As soon as $\Lambda \subset H_{0}$, the projection $\pi_{\Lambda}$ maps $\mathbb{A}^{n}=\mathbb{P}^{n} \backslash H_{0}$ into $\mathbb{A}^{m+1}$. Thus, let

$$
G_{0}=\left\{\Lambda \in \mathbb{G}(n-m-2, n) ; \Lambda \subset H_{0}\right\} \cong \mathbb{G}(n-m-2, n-1) .
$$

It is easy to see that

$$
\left(Y_{Z} \cup Y_{Z}^{\prime}\right) \cap G_{0} \subseteq Y_{Z_{0}} \cup Y_{Z_{0}}^{\prime},
$$

where $Z_{0}=Z \cap H_{0}$. Applying the arguments of [2], we deduce that $Y_{Z_{0}} \cup Y_{Z_{0}}^{\prime}$ is a proper closed subvariety of $\mathbb{G}(n-m-2, n-1)$ of degree $O_{n, d}(1)$. However, since $Z_{0}$ is not necessarily integral, this requires the following generalization of [2, Lemma 6], the proof of which is straightforward:

Lemma 6.1. Suppose that the closed subvariety $Z \subseteq \mathbb{P}^{n}$ is equidimensional of dimension $m$ and degree $d$. Then $Y_{Z}$ is a proper closed subvariety of $\mathbb{G}(n-m-2, n)$ of degree $O_{d, n}(1)$.

By [2, Lemma 3] we can therefore find an $(n-m-2)$-plane $\Lambda \notin Y_{Z_{0}} \cup Y_{Z_{0}}^{\prime}$ that is defined over $\mathbb{Q}$ and of bounded height. The projection $\pi_{\Lambda}: Z \rightarrow \mathbb{P}^{m+1}$ is then birational onto its image. Moreover, $\pi_{\Lambda}: Z_{0, i} \rightarrow \mathbb{P}^{m+1}$ is birational onto its image for each irreducible component $Z_{0, i}$ of $Z_{0}$.

Finally, choosing $\Gamma$ as explicitly described in [2], it is evident that $\pi_{\Lambda}$ maps integral points of height at most $B$ in $\mathbb{A}^{n}$ to integral points of height $O_{n, d}(B)$ in $\mathbb{A}^{m+1}$. This finishes our proof of Proposition 6.2 . 


\section{References}

[1] E. Ballico, An effective Bertini theorem over finite fields, Adv. Geom. 3 (2003), 361-363.

[2] T. D. Browning, D. R. Heath-Brown, and P. Salberger, Counting rational points on algebraic varieties, Duke Math. J. 132 (2006), 545-578.

[3] P. Deligne, La conjecture de Weil. I, Inst. Hautes Études Sci. Publ. Math. 43 (1974), 273-307.

[4] A. Grothendieck, Éléments de géométrie algébrique. III. Étude cohomologique des faisceaux cohérents. I, ibid. 11 (1961), 167 pp.

[5] —, Éléments de géométrie algébrique. IV. Étude locale des schémas et des morphismes de schémas. I, ibid. 20 (1964), 259 pp.

[6] —, Éléments de géométrie algébrique. IV. Étude locale des schémas et des morphismes de schémas. III, ibid. 28 (1966), $255 \mathrm{pp}$.

[7] G. H. Hardy, J. E. Littlewood, and G. Pólya, Inequalities, 2nd ed., Cambridge Univ. Press, Cambridge, 1952.

[8] G. H. Hardy and E. M. Wright, An Introduction to the Theory of Numbers, 4th ed., Clarendon Press, Oxford, 1960.

[9] J. Harris, Algebraic Geometry, Grad. Texts in Math. 133, Springer, New York, 1992.

[10] R. Hartshorne, Ample subvarieties of algebraic varieties, Lecture Notes in Math. 156, Springer, Berlin, 1970.

[11] —, Algebraic Geometry, Springer, New York, 1977.

[12] D. R. Heath-Brown, The density of rational points on nonsingular hypersurfaces, Proc. Indian Acad. Sci. Math. Sci. 104 (1994), 13-29.

[13] - The density of rational points on curves and surfaces, Ann. of Math. (2) 155 (2001), 553-595.

[14] C. Hooley, On the number of points on a complete intersection over a finite field, J. Number Theory 38 (1991), 338-358.

[15] J.-P. Jouanolou, Théorèmes de Bertini et Applications, Progr. Math. 42, Birkhäuser, Boston, 1983.

[16] N. M. Katz, Estimates for "singular" exponential sums, Int. Math. Res. Notices 1999, no. 16, 875-899.

[17] E. Kunz, Introduction to Commutative Algebra and Algebraic Geometry, Birkhäuser, Boston, 1985.

[18] Q. Liu, Algebraic Geometry and Arithmetic Curves, Oxford Grad. Texts in Math. 6, Oxford Univ. Press, Oxford, 2002.

[19] O. Marmon, The density of integral points on complete intersections, Q. J. Math. 59 (2008), 29-53.

[20] P. Salberger, Integral points on hypersurfaces of degree at least three, preprint.

[21] - Counting rational points on hypersurfaces of low dimension, Ann. Sci. École Norm. Sup. (4) 38 (2005), 93-115.

Oscar Marmon

Mathematical Sciences

Chalmers University of Technology

SE-412 96 Gothenburg, Sweden

E-mail: marmon@chalmers.se
Mathematical Sciences

University of Gothenburg SE-412 96 Gothenburg, Sweden

Received on 26.8.2008

and in revised form on 2.6.2009 